\title{
Search for Top-Quark Partners with Charge 5/3 in the Same-Sign Dilepton Final State
}

\author{
S. Chatrchyan et al. \\ (CMS Collaboration) \\ (Received 9 December 2013; published 30 April 2014)
}

\begin{abstract}
A search for the production of heavy partners of the top quark with charge $5 / 3$ is performed in events with a pair of same-sign leptons. The data sample corresponds to an integrated luminosity of $19.5 \mathrm{fb}^{-1}$ and was collected at $\sqrt{s}=8 \mathrm{TeV}$ by the CMS experiment. No significant excess is observed in the data above the expected background, and the existence of top-quark partners with masses below $800 \mathrm{GeV}$ is excluded at a $95 \%$ confidence level, assuming they decay exclusively to $t W$. This is the first limit on these particles from the LHC, and it is significantly more restrictive than previous limits.
\end{abstract}

DOI: 10.1103/PhysRevLett.112.171801

PACS numbers: 14.65.Jk, 12.60.-i, 13.85.Qk

Various extensions of the standard model (SM) address the hierarchy problem, caused by the quadratic divergences in the quantum-loop corrections to the Higgs boson mass, by proposing new heavy particles. Since the largest correction arises from the top-quark loop, a class of these models, based on composite Higgs scenarios [1-4], predicts the existence of heavy partners of the top quark to explain the cancellation of this correction. These "top-quark partners" are expected to have masses close to the electroweak symmetry breaking scale and thus would be accessible at the CERN Large Hadron Collider (LHC), located near Geneva, Switzerland. They may also have exotic charge ( $5 e / 3$, where $-e$ is the charge of the electron) and in this case would not contribute to the coupling of the Higgs boson to gluons [5]. Searches for such top-quark partners explore parameter space that is not excluded by the recent observation of a Higgs boson with properties consistent with those of the SM Higgs particle [6-14]. Theoretical predictions suggest that searches in the mass region from $500 \mathrm{GeV}$ to $1.5 \mathrm{TeV}$ present the greatest potential for discovery at the LHC $[2,15]$.

This Letter presents a search for exotic top-quark partners using LHC $p p$ collision data collected by the Compact Muon Solenoid (CMS) experiment at a center-ofmass energy $\sqrt{s}=8 \mathrm{TeV}$. The analysis is based on a data sample corresponding to an integrated luminosity of $19.5 \mathrm{fb}^{-1}$. We look for the $T_{5 / 3}$, an exotic top-quark partner with charge $5 e / 3$. We assume that the $T_{5 / 3}$ is pair produced via either gluon fusion or quark annihilation and decays via $T_{5 / 3} \rightarrow t W^{+}$followed by $t \rightarrow W^{+} b$ (charge conjugate modes are implied throughout this Letter). Single $T_{5 / 3}$ production is not considered because it is more model dependent and presents a different event topology [2].

* Full author list given at the end of the article.

Published by the American Physical Society under the terms of the Creative Commons Attribution 3.0 License. Further distribution of this work must maintain attribution to the author(s) and the published articles title, journal citation, and DOI.
We focus on the dilepton final state wherein, for one or both of the $T_{5 / 3}$, its two $W$ bosons both decay into leptons, which will have the same charge. Because of the presence of the two bottom quarks and the possibility of hadronic decays for one of the top-quark partners, this final state also includes significant jet activity. The leptons considered in this analysis are electrons and muons. The presence of leptons with the same electric charge (same-sign leptons) distinguishes this process from $t \bar{t}$, making the contribution of the latter comparable to backgrounds with much smaller cross sections: $t \bar{t} W, t \bar{t} W W, t \bar{t} Z, W W W$, and same-sign $W W$. Because of its large cross section, $t \bar{t}$ still contributes to the overall background through instrumental effects such as charge misidentification in dilepton decays, as well as through $t \bar{t}$ events where the $W$ boson from one top quark decays leptonically and the second lepton arises from a $b$-quark decay. Additional processes that contribute to the expected background include QCD multijets, $W / Z+$ jets, and dibosons ( $W Z$ and $Z Z$ ). A previous search using a signature of same-sign leptons, multiple jets, and missing transverse energy was performed by the CDF experiment and excludes $T_{5 / 3}$ masses below $365 \mathrm{GeV}$ at the $95 \%$ confidence level (C.L.) [16]. The CDF Collaboration also set a limit on the production of exotic quarks with charge $-4 e / 3$ [17].

The central feature of the CMS apparatus is a superconducting solenoid of $6 \mathrm{~m}$ internal diameter, providing a magnetic field of $3.8 \mathrm{~T}$. Within the superconducting solenoid volume are a silicon pixel and strip tracker, a lead tungstate crystal electromagnetic calorimeter (ECAL), and a brass and scintillator hadron calorimeter. Muons are measured in gas-ionization detectors embedded in the steel flux return yoke outside the solenoid. In addition, the CMS detector has extensive forward calorimetry. A more detailed description of the CMS detector can be found elsewhere [18].

Simulation of the pair production of top-quark partners was performed with the MADGRAPH 5.1.1 [19] event generator, and 12 samples corresponding to values of the 
$T_{5 / 3}$ mass from $350 \mathrm{GeV}$ to $1 \mathrm{TeV}$ were produced. The PYTHIA 6.426 [20] generator was used for parton showering, hadronization, and simulation of the underlying event. The CTEQ6L [21] parton distribution functions were used, and the PYTHIA parameters for the underlying event were set to the $Z 2^{*}$ [22] tune. The detector response was modeled using GEANT4 [23]. The next-to-next-to-leading-order cross section for $T_{5 / 3}$ pair production was found using TOP++ [24] to vary from $5.3 \mathrm{pb}$ at the mass of $350 \mathrm{GeV}$ to $3.4 \mathrm{fb}$ at the mass of $1 \mathrm{TeV}$. The uncertainty on the cross section in the mass range used for the analysis is about $5 \%$.

The background processes include $t \bar{t}, W(\rightarrow \ell \nu)+$ jets, $Z / \gamma^{*}(\rightarrow \ell \ell)+$ jets, and dibosons (WZ and $Z Z$ ). Additional low-rate $\mathrm{SM}$ processes were also considered: $W^{ \pm} W^{ \pm}, W W W, t \bar{t} W, t \bar{t} W W$, and $t \bar{t} Z$. These were all simulated by means of MADGRAPH with PYTHIA used for hadronization. Next-to-leading-order or, where available, next-to-next-to-leading-order cross sections were employed $[25,26]$.

For all the simulated samples, the additional proton-proton interactions in each beam crossing (pileup) were modeled by superimposing minimum bias interactions (obtained using PYTHIA with the $Z 2^{*}$ tune) onto simulated events, with the multiplicity distribution matching the one observed in data.

This analysis relies on the reconstruction of three physical objects: electrons, muons, and jets. The events are reconstructed using the CMS "particle-flow" event description algorithm [27,28]. Candidate events are required to have at least two leptons with the same charge that are within the detector acceptance $(|\eta|<2.4)$ and to have passed triggers based on dielectrons, dimuons, or electron-muon combinations. Here, $\eta$ is the pseudorapidity defined as $\eta \equiv-\ln [\tan (\theta / 2)]$, where $\theta$ is the polar angle with respect to the counterclockwise beam direction. Candidate events must also have at least one good reconstructed primary vertex matched to the track origins of the two selected leptons. Candidate electrons are reconstructed using energy deposits in the ECAL and a track from the silicon detectors. The shape of the shower in the ECAL must be consistent with that of an electron, and the shower must be well matched to the extrapolated track. Additional requirements are imposed to reject electrons produced by photon conversions. The charge of each electron candidate is measured using three different methods. Two of the measurements are based on two different tracking algorithms: the standard CMS track reconstruction algorithm [29] and the Gaussian sum filter algorithm [30], optimized to take into account the possible emission of bremsstrahlung photons in the silicon tracker. The third measurement is based on the relative position of the calorimeter cluster and the projected track from the pixel detector. All three measurements are required to be consistent with the electron hypothesis. Muon candidates are required to be reconstructed by both the silicon tracker and the muon system, and the combined fit of the track must be of good quality $\left(\chi^{2}\right.$ per degree of freedom less than 10$)$.
All selected leptons are required to be isolated. The isolation for each lepton is estimated by first computing the scalar sum of the transverse momenta of all neutral and charged reconstructed particle candidates, except the lepton itself, within a cone of size $\Delta R \equiv \sqrt{(\Delta \phi)^{2}+(\Delta \eta)^{2}}$ around the lepton, where $\phi$ is the azimuthal angle. This sum is then divided by the transverse momentum $\left(p_{T}\right)$ of the lepton to calculate the relative isolation $\left(I_{R}\right)$. The values for the cone size and the maximum allowed $I_{R}$ are $\Delta R=0.3(0.4)$ and $I_{R}=0.15(0.20)$, respectively, for electrons (muons), as determined by optimization studies for CMS top-quark related analyses. An event-by-event correction is applied to the computation of the lepton isolation in order to account for the effect of pileup. Scale factors to correct for imperfect detector simulation are obtained using the tag-and-probe method [31] for lepton identification and isolation as a function of lepton $p_{T}$ and $\eta$. In addition, we define a category of "loose" leptons with some of the isolation and identification requirements relaxed. The $I_{R}$ threshold for these leptons is increased from 0.15 to 0.60 for electrons and from 0.20 to 0.40 for muons.

For the range of $T_{5 / 3}$ masses accessible at $\sqrt{s}=8 \mathrm{TeV}$, the analysis exploits advanced techniques in jet reconstruction for identifying highly boosted top quarks and $W$ bosons that decay hadronically. In particular, if the top quarks are highly boosted $\left(p_{T}>400 \mathrm{GeV}\right)$, their decay products are collimated and merged into one jet. We use a "top-quark tagging" algorithm based on identifying jet substructure [32] to reconstruct such merged top-quark jets. Jets are clustered using the Cambridge-Aachen algorithm [33,34], as implemented in FASTJET version 3 [35], with a distance parameter of $R=0.8$ in $\eta-\phi$ space (CA 8 jets). The CA8 top-quark jets are required to have $p_{T}>400 \mathrm{GeV}$ and more than two subjets found by the top-quark tagging algorithm. The jet mass must be consistent with the mass of the top quark, and the minimum pairwise mass of the three highest $p_{T}$ subjets is required to be greater than $50 \mathrm{GeV}$.

The decay products of $W$ bosons from the $T_{5 / 3}$ decay or from a highly boosted top quark, for which the $b$ quark is reconstructed independently, may also merge into a single jet. We use a "jet pruning" algorithm [36] to identify the hadronic decay of such $W$ bosons. This algorithm also uses CA8 jets as inputs with the pruning parameters taken from the original theoretical papers $[37,38]$. CA8 $W$-boson jets are required to have $p_{T}>200 \mathrm{GeV}$, exactly two subjets, and their mass must be consistent with that of the $W$ boson [39].

To account for $W$ bosons and top quarks that are not highly boosted, jets are also reconstructed using the anti- $k_{T}$ algorithm [40] with a distance parameter of 0.5 (AK5). These jets are required to have $p_{T}>30 \mathrm{GeV}$. If an AK5 jet overlaps with a top-quark jet or a W-boson jet $(\Delta R<0.8)$, the AK5 jet is discarded.

All of the above categories of jets are required to have $|\eta|<2.4$ and particle-flow jet identification [41]. Jet energy 
corrections are applied to account for residual nonuniformity and nonlinearity of the detector response. Jet energies are also corrected by subtracting the average contribution of particles from pileup $[42,43]$. For the simulated samples, additional smearing is applied to the jet $p_{T}(\sim 7 \%-19 \%$ depending on $\eta$ ) in order to reproduce the jet energy resolution observed in data. All jets must be $\Delta R \geq 0.3$ away from the selected leptons and, as mentioned above, $\Delta R \geq 0.8$ away from any other jet. A correction to account for differences in the identification efficiency of $W$-boson and top-quark jets between data and simulation is applied [44].

The signal selection, optimized to yield the best signal sensitivity, requires the following. (i) At least two isolated same-sign leptons as defined above with $p_{T}>30 \mathrm{GeV}$. Between each lepton and every top-quark jet, we require $\Delta R>0.8$. (ii) Dilepton Z-boson veto: $M(e e)<76 \mathrm{GeV}$ or $M(e e)>106 \mathrm{GeV}$. This selection applies only to the dielectron channel. If the muon charge is mismeasured, its momentum will also be mismeasured, so a selected muon pair from a $Z$ boson will not fall within this invariant mass range. (iii) Trilepton $Z$-boson veto: $M(\ell \ell)<76 \mathrm{GeV}$ or $M(\ell \ell)>106 \mathrm{GeV}$, where $M(\ell \ell)$ is the invariant mass of either one of the selected leptons and any other sameflavor opposite-sign lepton in the event with $p_{T}>15 \mathrm{GeV}$ that satisfies the loose lepton criteria. (iv) $N_{c} \geq 7$, where $N_{c}$ is the number of constituents identified in the event. For the purpose of this selection, each AK5 jet and each lepton count as one constituent. Since a $W$-boson jet is assumed to correspond to a $W$ boson, each such jet counts as two constituents, corresponding to the $W$-boson decay products. Likewise, each top-quark jet represents a top quark and counts as three constituents. (v) $H_{T}>900 \mathrm{GeV}$, where $H_{T}$ is the scalar sum of the $p_{T}$ of all selected jets and leptons in the event. With these criteria, the signal efficiency is $10 \%-13 \%$ for $T_{5 / 3}$ masses between 750 and $1000 \mathrm{GeV}$.

The backgrounds associated with this analysis fall into three main categories. First, they may originate from SM processes leading to prompt, same-sign dilepton signatures, including diboson production ( $W Z$ and $Z Z$ ), $t \bar{t} W, t \bar{t} W W, t \bar{t} Z, W^{ \pm} W^{ \pm}$, and $W W W$. The contribution of these backgrounds is obtained from simulation.

The second category consists of events from processes with prompt, opposite-sign leptons, such as $t \bar{t}$ and DrellYan production, in which one of the leptons is misreconstructed with the wrong charge, leading to a same-sign dilepton final state. For muons in the $p_{T}$ range typical of the dominant backgrounds, the charge misidentification rate is extremely small (of order $10^{-4}$ ) and its contribution to the background is negligible [45]. For electrons, the charge misidentification probability $\left(\sim 10^{-3}\right)$ is derived from a data sample dominated by Drell-Yan events obtained by selecting dileptons with an invariant mass consistent with originating from the $Z$ boson, using the ratio of same-sign
Z-boson candidates to the total number of candidates. The number of expected same-sign events due to charge misidentification is then estimated by considering the total number of events passing the full selection but having oppositely charged leptons. These events are weighted by the charge misidentification probability parametrized as a function of the electron $p_{T}$ and $\eta$ to obtain the contribution of this background type.

The third category consists of events with one or more "nonprompt leptons." This is the primary instrumental background arising from jets being misidentified as leptons and nonprompt leptons passing tight isolation selection criteria. This contribution is estimated using the "tightloose" method described in Ref. [46]. "Tight" leptons have the same definition as those used in the analysis, whereas "loose" leptons are defined earlier. The background is estimated by using events with one or more loose leptons weighted by the ratios of the numbers of tight leptons to the numbers of loose leptons expected for prompt and nonprompt leptons. The ratio for prompt leptons is determined from Drell-Yan events where the invariant mass of the leptons is within $10 \mathrm{GeV}$ of the $Z$-boson mass. The nonprompt ratio is determined from a sample enriched in background by requiring exactly one lepton, low missing transverse energy $\left(E_{T}^{\text {miss }}<25 \mathrm{GeV}\right)$, low transverse mass $\left(M_{T}<25 \mathrm{GeV}\right)$, and at least one jet (the "away jet") with $p_{T}>40 \mathrm{GeV}$ and $\Delta R>1.0$ with respect to the lepton. The transverse mass is defined as $M_{T} \equiv \sqrt{2 p_{T}^{\ell} E_{T}^{\text {miss }}(1-\cos \Delta \phi)}$, where $\Delta \phi$ is the angle between the lepton transverse momentum $\left(p_{T}^{\ell}\right)$ and the direction associated with $E_{T}^{\text {miss }}$.

The systematic uncertainties that affect the signal and background acceptance include uncertainties in the efficiency of the trigger (1\%), lepton reconstruction and identification efficiency ( $1 \%$ per lepton), pileup, and the jet energy scale (JES). The uncertainties due to the JES and pileup are obtained by varying the respective quantities in simulation. For the signal, the JES and pileup uncertainties in the acceptance correspond to $2 \%$ and $3 \%$, respectively. For the simulated backgrounds, they range from $3 \%$ to $6 \%$, depending on the sample. In addition, we assign a constant $3 \%$ uncertainty due to the JES of CA8 jets for all simulated samples [44]. The dominant uncertainty in the expected event yields due to backgrounds derived from simulation is the overall normalization uncertainty. The $Z Z(5.1 \%), W Z$ $(17 \%)$, and $t \bar{t} W(32 \%)$ normalization uncertainties are taken from Refs. [26,47,48], respectively. For the other rare backgrounds, we assume a conservative normalization uncertainty of 50\% [49]. An uncertainty of $20 \%$ is assigned to the background contribution from charge misidentification, based on the difference in the charge misidentification rate between Drell-Yan data and $t \bar{t}$ simulation. Following Ref. [45], we also assign a conservative additional systematic uncertainty of $50 \%$ in the estimation of backgrounds due to nonprompt leptons. This uncertainty is 
TABLE I. Summary table of expected and observed numbers of events for all channels. The background is composed of the same-sign component, the contribution due to charge misidentification, and that due to misreconstructed leptons. All systematic uncertainties are included. Also shown is the expected contribution from a $T_{5 / 3}$ with mass of $800 \mathrm{GeV}$.

\begin{tabular}{lcccc}
\hline \hline Channel & $e e$ & $e \mu$ & $\mu \mu$ & All \\
\hline Same sign & $0.8 \pm 0.2$ & $1.9 \pm 0.4$ & $1.3 \pm 0.3$ & $4.0 \pm 0.8$ \\
Charge misidentification & $0.06 \pm 0.02$ & $0.04 \pm 0.01$ & $\ldots$ & $0.11 \pm 0.02$ \\
Nonprompt & $1.9 \pm 1.2$ & $0.6 \pm 0.9$ & $0.3 \pm 0.6$ & $2.8 \pm 1.9$ \\
Total background & $2.7 \pm 1.3$ & $2.5 \pm 1.0$ & $1.6 \pm 0.7$ & 3 \\
Observed events & 0 & 6 & $2.8 \pm 0.2$ & 9 \\
$T_{5 / 3}$ & $2.1 \pm 0.1$ & $4.7 \pm 0.3$ & $9.7 \pm 0.5$ \\
\hline \hline
\end{tabular}

based on closure tests using $t \bar{t}$ and $W+$ jets simulated samples and takes into account variations due to the away jet $p_{T}$ and the flavor composition of the background, thus also accounting for any potential dependence on kinematic parameters that alter the background composition (such as $\left.H_{T}\right)$. We also include a $2.6 \%$ uncertainty due to the luminosity [50] for all event yields that are derived from simulation.

The final numbers of observed and expected events are reported in Table I for each of the three lepton channels $(e e, e \mu$, and $\mu \mu$ ) and their combination. Figure 1 shows the $H_{T}$ distribution for all channels combined.

No significant excess is observed. Exclusion limits are computed at $95 \%$ C.L. by using the ROOSTATS implementation [51] of the Bayesian approach. We use

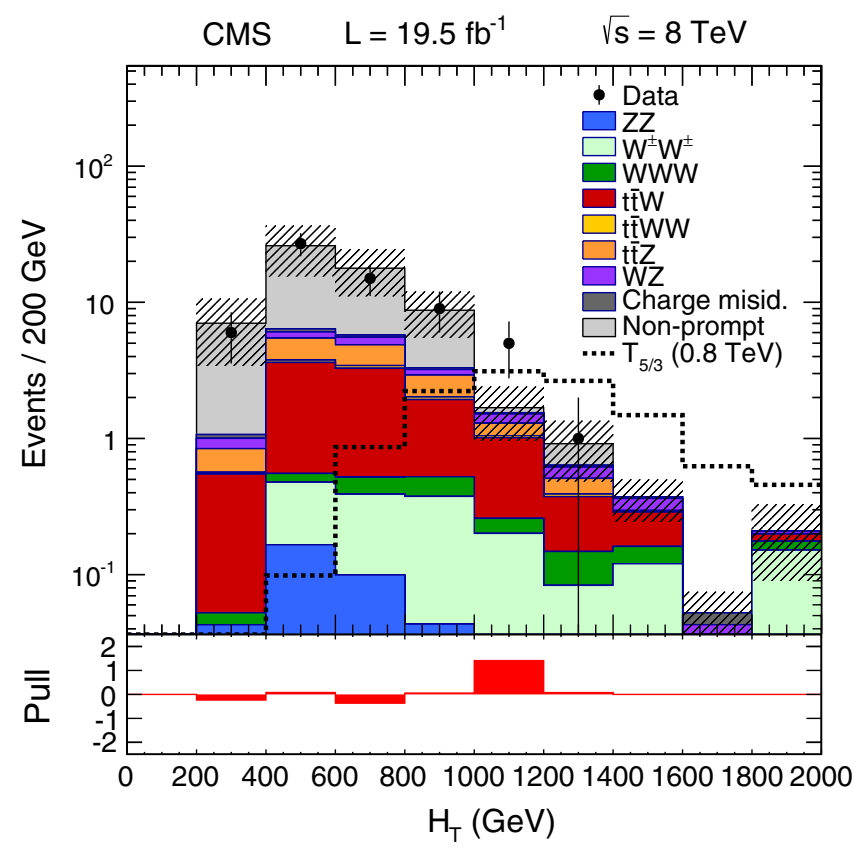

FIG. 1 (color online). The distribution of $H_{T}$ for all channels combined after the full selection except for the $H_{T}$ requirement itself. The shaded band represents the total uncertainty in the predicted backgrounds. The final bin includes all overflow events. The pull is defined as the difference between the observed and expected values divided by the total uncertainty. a cut-and-count method and compare the numbers of observed events with the numbers of expected signal and background events. A flat prior is used for the signal production cross section. The event yields from all lepton channels are combined when setting the limits. Upper bounds are set on the production cross section of heavy top-quark partners, assuming a $100 \%$ branching fraction $(\mathrm{BF})$ for the decay $T_{5 / 3} \rightarrow t W$. The resulting expected and observed limits are shown in Fig. 2. The expected lower limit on the mass of the $T_{5 / 3}$ is $830 \mathrm{GeV}$, and the observed limit is $800 \mathrm{GeV}$.

The use of recently developed jet substructure techniques in this analysis for identifying boosted top quarks and $\mathrm{W}$ bosons enables us to probe cross sections of $T_{5 / 3}$ pair production that are between 10\%-20\% lower than would otherwise be possible for $T_{5 / 3}$ masses in the range $800-$ $1000 \mathrm{GeV}$. The reconstruction of the $T_{5 / 3}$ mass benefits as well, and this can, in the event of a discovery in the future,

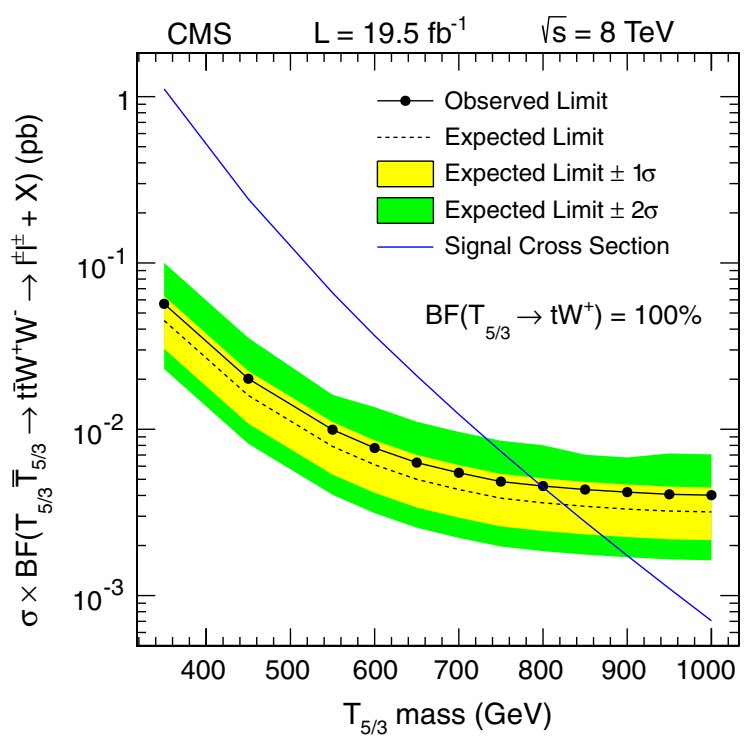

FIG. 2 (color online). Expected and observed 95\% C.L. limits on the $T_{5 / 3}$ production cross section times the branching fraction for decay to same-sign dileptons. The $1 \sigma$ and $2 \sigma$ combined statistical and systematic expected variations are shown as yellow (light) and green (dark) bands, respectively. 
be used to distinguish a $T_{5 / 3}$ from other exotic particles which decay in a similar manner [52].

In summary, a search for an exotic top partner with charge $5 / 3$ in same-sign dileptonic events has been performed using $19.5 \mathrm{fb}^{-1}$ of data collected by the CMS experiment at $\sqrt{s}=8 \mathrm{TeV}$. No significant excess is observed in the data above the expected standard model background. An upper bound at the 95\% confidence level is set on the production cross section of heavy top-quark partners, assuming a $100 \%$ branching fraction for the decay $T_{5 / 3} \rightarrow t W$, and masses below $800 \mathrm{GeV}$ are excluded. This is the first limit on $T_{5 / 3}$ production from the $\mathrm{LHC}$, and it is significantly more restrictive than the $365 \mathrm{GeV}$ limit set by previous searches.

We congratulate our colleagues in the CERN accelerator departments for the excellent performance of the LHC and thank the technical and administrative staffs at CERN and at other CMS institutes for their contributions to the success of the CMS effort. In addition, we gratefully acknowledge the computing centers and personnel of the Worldwide LHC Computing Grid for delivering so effectively the computing infrastructure essential to our analyses. Finally, we acknowledge the enduring support for the construction and operation of the LHC and the CMS detector provided by the following funding agencies: BMWF and FWF (Austria); FNRS and FWO (Belgium); CNPq, CAPES, FAPERJ, and FAPESP (Brazil); MES (Bulgaria); CERN; CAS, MoST, and NSFC (China); COLCIENCIAS (Colombia); MSES (Croatia); RPF (Cyprus); MoER, SF0690030s09, and ERDF (Estonia); Academy of Finland, MEC, and HIP (Finland); CEA and CNRS/ IN2P3 (France); BMBF, DFG, and HGF (Germany); GSRT (Greece); OTKA and NKTH (Hungary); DAE and DST (India); IPM (Iran); SFI (Ireland); INFN (Italy); NRF and WCU (Republic of Korea); LAS (Lithuania); CINVESTAV, CONACYT, SEP, and UASLP-FAI (Mexico); MSI (New Zealand); PAEC (Pakistan); MSHE and NSC (Poland); FCT (Portugal); JINR (Dubna); MON, RosAtom, RAS, and RFBR (Russia); MESTD (Serbia); SEIDI and CPAN (Spain); Swiss Funding Agencies (Switzerland); NSC (Taipei); ThEPCenter, IPST, STAR, and NSTDA (Thailand); TUBITAK and TAEK (Turkey); NASU (Ukraine); STFC (United Kingdom); and DOE and NSF (U.S.A.).

[1] A. De Simone, O. Matsedonskyi, R. Rattazzi, and A. Wulzer, J. High Energy Phys. 04 (2013) 004.

[2] J. Mrazek and A. Wulzer, Phys. Rev. D 81, 075006 (2010).

[3] G. Dissertori, E. Furlan, F. Moortgat, and P. Nef, J. High Energy Phys. 09 (2010) 019.

[4] R. Contino and G. Servant, J. High Energy Phys. 06 (2008) 026.

[5] A. Azatov and J. Galloway, Phys. Rev. D 85, 055013 (2012).
[6] ATLAS Collaboration, Phys. Lett. B 716, 1 (2012).

[7] CMS Collaboration, Phys. Lett. B 716, 30 (2012).

[8] CMS Collaboration, J. High Energy Phys. 06 (2013) 081.

[9] ATLAS Collaboration, Phys. Lett. B 726, 88 (2013).

[10] ATLAS Collaboration, Phys. Lett. B 726, 120 (2013).

[11] CMS Collaboration, Phys. Rev. Lett. 110, 081803 (2013).

[12] CMS Collaboration, J. High Energy Phys. 01 (2014) 096.

[13] CMS Collaboration, arXiv:1312.5353.

[14] CMS Collaboration, arXiv:1401.6527.

[15] R. Contino, L. Da Rold, and A. Pomarol, Phys. Rev. D 75, 055014 (2007).

[16] T. Aaltonen et al. (CDF Collaboration), Phys. Rev. Lett. 104, 091801 (2010).

[17] T. Aaltonen et al. (CDF Collaboration), Phys. Rev. Lett. 105, 101801 (2010).

[18] CMS Collaboration, JINST 3, S08004 (2008).

[19] J. Alwall, M. Herquet, F. Maltoni, O. Mattelaer, and T. Stelzer, J. High Energy Phys. 06 (2011) 128.

[20] T. Sjöstrand, S. Mrenna, and P. Z. Skands, J. High Energy Phys. 05 (2006) 026.

[21] J. Pumplin, D. R. Stump, J. Huston, H.-L. Lai, P. Nadolsky, and W.-K. Tung, J. High Energy Phys. 07 (2002) 012.

[22] CMS Collaboration, J. High Energy Phys. 09 (2011) 109.

[23] S. Agostinelli et al. (GEANT4 Collaboration), Nucl. Instrum. Methods Phys. Res., Sect. A 506, 250 (2003).

[24] M. Czakon, P. Fiedler, and A. Mitov, Phys. Rev. Lett. 110, 252004 (2013).

[25] M. V. Garzelli, A. Kardos, C. G. Papadopoulos, and Z. Trocsanyi, J. High Energy Phys. 11 (2012) 056.

[26] J. M. Campbell, R. K. Ellis, and C. Williams, J. High Energy Phys. 07 (2011) 018.

[27] CMS Collaboration, CMS Physics Analysis Summary Report No. CMS-PAS-PFT-10-003, 2010.

[28] CMS Collaboration, CMS Physics Analysis Summary Report No. CMS-PAS-PFT-09-001, 2009.

[29] CMS Collaboration, CMS Physics Analysis Summary Report No. CMS-PAS-TRK-10-001, 2010.

[30] W. Adam, R. Frühwirth, A. Strandlie, and T. Todorov, J. Phys. G 31, N9 (2005).

[31] CMS Collaboration, J. High Energy Phys. 10 (2011) 132.

[32] CMS Collaboration, CMS Physics Analysis Summary Report No. CMS-PAS-JME-09-001, 2009.

[33] M. Wobisch and T. Wengler, arXiv:hep-ph/9907280.

[34] Y. L. Dokshitzer, G. D. Leder, S. Moretti, and B. R. Webber, J. High Energy Phys. 08 (1997) 001.

[35] M. Cacciari, G. P. Salam, and G. Soyez, Eur. Phys. J. C 72, 1896 (2012).

[36] CMS Collaboration, CMS Physics Analysis Summary Report No. CMS-PAS-JME-10-013, 2010.

[37] S. D. Ellis, C. K. Vermilion, and J. R. Walsh, Phys. Rev. D 80, 051501 (2009).

[38] S. D. Ellis, C. K. Vermilion, and J. R. Walsh, Phys. Rev. D 81, 094023 (2010).

[39] CMS Collaboration, CMS Physics Analysis Summary Report No. CMS-PAS-JME-13-006, 2013.

[40] M. Cacciari, G. P. Salam, and G. Soyez, J. High Energy Phys. 04 (2008) 063.

[41] CMS Collaboration, J. Instrum. 6, P11002 (2011).

[42] M. Cacciari and G. P. Salam, Phys. Lett. B 659, 119 (2008). 
[43] M. Cacciari, G. P. Salam, and G. Soyez, J. High Energy Phys. 04 (2008) 005.

[44] CMS Collaboration, Phys. Rev. Lett. 111, 211804 (2013).

[45] CMS Collaboration, J. High Energy Phys. 08 (2012) 110.

[46] CMS Collaboration, J. High Energy Phys. 06 (2011) 077.

[47] ATLAS Collaboration, Phys. Lett. B 709, 341 (2012).

[48] J. M. Campbell and R. K. Ellis, J. High Energy Phys. 07 (2012) 052.

[49] CMS Collaboration, J. High Energy Phys. 03 (2013) 037; CMS Collaboration, J. High Energy Phys. 07 (2013) 041.
[50] CMS Collaboration, CMS Physics Analysis Summary Report No. CMS-PAS-LUM-13-001, 2013.

[51] L. Moneta, K. Belasco, K. S. Cranmer, A. Lazzaro, D. Piparo, G. Schott, W. Verkerke, and M. Wolf, Proc. Sci., ACAT2010 (2010) 057 [arXiv:1009.1003].

[52] See Supplemental Material at http://link.aps.org/ supplemental/10.1103/PhysRevLett.112.171801 for figures illustrating the agreement of data with background predictions and showing the reconstructed top partner mass.

S. Chatrchyan, ${ }^{1}$ V. Khachatryan, ${ }^{1}$ A. M. Sirunyan, ${ }^{1}$ A. Tumasyan, ${ }^{1}$ W. Adam, ${ }^{2}$ T. Bergauer, ${ }^{2}$ M. Dragicevic, ${ }^{2}$ J. Erö, ${ }^{2}$ C. Fabjan, ${ }^{2, b}$ M. Friedl, ${ }^{2}$ R. Frühwirth ${ }^{2, b}$ V. M. Ghete, ${ }^{2}$ C. Hartl, ${ }^{2}$ N. Hörmann, ${ }^{2}$ J. Hrubec, ${ }^{2}$ M. Jeitler, ${ }^{2, b}$ W. Kiesenhofer, ${ }^{2}$ V. Knünz, ${ }^{2}$ M. Krammer, ${ }^{2, b}$ I. Krätschmer, ${ }^{2}$ D. Liko, ${ }^{2}$ I. Mikulec, ${ }^{2}$ D. Rabady, ${ }^{2, c}$ B. Rahbaran, ${ }^{2}$ H. Rohringer, ${ }^{2}$

R. Schöfbeck, ${ }^{2}$ J. Strauss, ${ }^{2}$ A. Taurok, ${ }^{2}$ W. Treberer-Treberspurg, ${ }^{2}$ W. Waltenberger, ${ }^{2}$ C.-E. Wulz, ${ }^{2, b}$ V. Mossolov, ${ }^{3}$ N. Shumeiko, ${ }^{3}$ J. Suarez Gonzalez, ${ }^{3}$ S. Alderweireldt, ${ }^{4}$ M. Bansal ${ }^{4}{ }^{4}$. Bansal, ${ }^{4}$ T. Cornelis, ${ }^{4}$ E. A. De Wolf, ${ }^{4}$ X. Janssen, ${ }^{4}$ A. Knutsson, ${ }^{4}$ S. Luyckx, ${ }^{4}$ L. Mucibello, ${ }^{4}$ S. Ochesanu, ${ }^{4}$ B. Roland, ${ }^{4}$ R. Rougny, ${ }^{4}$ H. Van Haevermaet, ${ }^{4}$ P. Van Mechelen, ${ }^{4}$ N. Van Remortel, ${ }^{4}$ A. Van Spilbeeck, ${ }^{4}$ F. Blekman, ${ }^{5}$ S. Blyweert, ${ }^{5}$ J. D'Hondt, ${ }^{5}$ N. Heracleous, ${ }^{5}$ A. Kalogeropoulos, ${ }^{5}$

J. Keaveney, ${ }^{5}$ T. J. Kim, ${ }^{5}$ S. Lowette, ${ }^{5}$ M. Maes, ${ }^{5}$ A. Olbrechts, ${ }^{5}$ D. Strom,${ }^{5}$ S. Tavernier, ${ }^{5}$ W. Van Doninck, ${ }^{5}$ P. Van Mulders, ${ }^{5}$ G. P. Van Onsem, ${ }^{5}$ I. Villella, ${ }^{5}$ C. Caillol, ${ }^{6}$ B. Clerbaux, ${ }^{6}$ G. De Lentdecker, ${ }^{6}$ L. Favart, ${ }^{6}$ A. P. R. Gay, ${ }^{6}$ T. Hreus, ${ }^{6}$ A. Léonard, ${ }^{6}$ P. E. Marage, ${ }^{6}$ A. Mohammadi, ${ }^{6}$ L. Perniè, ${ }^{6}$ T. Reis,${ }^{6}$ T. Seva, ${ }^{6}$ L. Thomas, ${ }^{6}$ C. Vander Velde, ${ }^{6}$ P. Vanlaer, ${ }^{6}$ J. Wang, ${ }^{6}$ V. Adler, ${ }^{7}$ K. Beernaert, ${ }^{7}$ L. Benucci, ${ }^{7}$ A. Cimmino, ${ }^{7}$ S. Costantini, ${ }^{7}$ S. Dildick, ${ }^{7}$ G. Garcia, ${ }^{7}$ B. Klein, ${ }^{7}$ J. Lellouch, ${ }^{7}$ J. Mccartin, ${ }^{7}$ A. A. Ocampo Rios, ${ }^{7}$ D. Ryckbosch, ${ }^{7}$ M. Sigamani, ${ }^{7}$ N. Strobbe, ${ }^{7}$ F. Thyssen, ${ }^{7}$ M. Tytgat, ${ }^{7}$ S. Walsh, ${ }^{7}$ E. Yazgan, ${ }^{7}$ N. Zaganidis, ${ }^{7}$ S. Basegmez, ${ }^{8}$ C. Beluffi,${ }^{8, d}$ G. Bruno, ${ }^{8}$ R. Castello, ${ }^{8}$ A. Caudron, ${ }^{8}$ L. Ceard, ${ }^{8}$ G. G. Da Silveira, ${ }^{8}$ C. Delaere, ${ }^{8}$ T. du Pree, ${ }^{8}$ D. Favart, ${ }^{8}$ L. Forthomme, ${ }^{8}$ A. Giammanco, ${ }^{8, e}$ J. Hollar, ${ }^{8}$ P. Jez, ${ }^{8}$ M. Komm, ${ }^{8}$ V. Lemaitre, ${ }^{8}$ J. Liao, ${ }^{8}$ O. Militaru, ${ }^{8}$ C. Nuttens, ${ }^{8}$ D. Pagano, ${ }^{8}$ A. Pin, ${ }^{8}$ K. Piotrzkowski, ${ }^{8}$ A. Popov,,${ }^{8, \mathrm{f}}$ L. Quertenmont, ${ }^{8}$ M. Selvaggi, ${ }^{8}$ M. Vidal Marono, ${ }^{8}$ J. M. Vizan Garcia, ${ }^{8}$ N. Beliy, ${ }^{9}$ T. Caebergs, ${ }^{9}$ E. Daubie, ${ }^{9}$ G. H. Hammad, ${ }^{9}$ G. A. Alves,${ }^{10}$ M. Correa Martins Junior, ${ }^{10}$ T. Martins, ${ }^{10}$ M. E. Pol, ${ }^{10}$ M. H. G. Souza, ${ }^{10}$ W. L. Aldá Júnior, ${ }^{11}$ W. Carvalho, ${ }^{11}$ J. Chinellato, ${ }^{11, \mathrm{~g}}$ A. Custódio, ${ }^{11}$ E. M. Da Costa, ${ }^{11}$ D. De Jesus Damiao, ${ }^{11}$ C. De Oliveira Martins,${ }^{11}$ S. Fonseca De Souza, ${ }^{11}$ H. Malbouisson, ${ }^{11}$ M. Malek, ${ }^{11}$ D. Matos Figueiredo, ${ }^{11}$ L. Mundim, ${ }^{11}$ H. Nogima, ${ }^{11}$ W. L. Prado Da Silva, ${ }^{11}$ J. Santaolalla,${ }^{11}$ A. Santoro, ${ }^{11}$ A. Sznajder, ${ }^{11}$ E. J. Tonelli Manganote, ${ }^{11, g}$ A. Vilela Pereira,${ }^{11}$ C. A. Bernardes, ${ }^{12 b}$ F. A. Dias, ${ }^{12 a, h}$ T. R. Fernandez Perez Tomei, ${ }^{12 a}$ E. M. Gregores, ${ }^{12 b}$ C. Lagana, ${ }^{12 a}$ P. G. Mercadante, ${ }^{12 b}$ S. F. Novaes, ${ }^{12 a}$ Sandra S. Padula, ${ }^{12 a}$ V. Genchev, ${ }^{13, \mathrm{c}}$ P. Iaydjiev, ${ }^{13, c}$ A. Marinov, ${ }^{13}$ S. Piperov, ${ }^{13}$ M. Rodozov, ${ }^{13}$ G. Sultanov, ${ }^{13}$ M. Vutova, ${ }^{13}$ A. Dimitrov, ${ }^{14}$ I. Glushkov, ${ }^{14}$ R. Hadjiiska, ${ }^{14}$ V. Kozhuharov,${ }^{14}$ L. Litov ${ }^{14}$ B. Pavlov, ${ }^{14}$ P. Petkov, ${ }^{14}$ J. G. Bian, ${ }^{15}$ G. M. Chen, ${ }^{15}$ H. S. Chen, ${ }^{15}$ M. Chen, ${ }^{15}$ R. Du, ${ }^{15}$ C. H. Jiang, ${ }^{15}$ D. Liang, ${ }^{15}$ S. Liang, ${ }^{15}$ X. Meng, ${ }^{15}$ R. Plestina, ${ }^{15, i}$ J. Tao, ${ }^{15}$ X. Wang, ${ }^{15}$ Z. Wang ${ }^{15}$ C. Asawatangtrakuldee, ${ }^{16}$ Y. Ban, ${ }^{16}$ Y. Guo, ${ }^{16}$ Q. Li ${ }^{16}$ W. Li,${ }^{16}$ S. Liu, ${ }^{16}$ Y. Mao, ${ }^{16}$ S. J. Qian, ${ }^{16}$ D. Wang,${ }^{16}$ L. Zhang, ${ }^{16}$ W. Zou, ${ }^{16}$ C. Avila, ${ }^{17}$ C. A. Carrillo Montoya, ${ }^{17}$ L. F. Chaparro Sierra, ${ }^{17}$ C. Florez,${ }^{17}$ J. P. Gomez,${ }^{17}$ B. Gomez Moreno, ${ }^{17}$ J. C. Sanabria, ${ }^{17}$ N. Godinovic, ${ }^{18}$ D. Lelas, ${ }^{18}$ D. Polic, ${ }^{18}$ I. Puljak, ${ }^{18}$ Z. Antunovic, ${ }^{19}$ M. Kovac, ${ }^{19}$ V. Brigljevic, ${ }^{20}$ K. Kadija, ${ }^{20}$ J. Luetic, ${ }^{20}$ D. Mekterovic,${ }^{20}$ S. Morovic,${ }^{20}$ L. Tikvica, ${ }^{20}$ A. Attikis, ${ }^{21}$ G. Mavromanolakis,${ }^{21}$ J. Mousa, ${ }^{21}$ C. Nicolaou, ${ }^{21}$ F. Ptochos, ${ }^{21}$ P. A. Razis, ${ }^{21}$ M. Finger, ${ }^{22}$ M. Finger Jr., ${ }^{22}$ A. A. Abdelalim, ${ }^{23, j}$ Y. Assran, ${ }^{23, k}$ S. Elgammal, ${ }^{23, j}$ A. Ellithi Kamel, ${ }^{23,1}$ M. A. Mahmoud ${ }^{23, m}$ A. Radi, ${ }^{23, n, o}$ M. Kadastik, ${ }^{24}$ M. Müntel, ${ }^{24}$ M. Murumaa, ${ }^{24}$ M. Raidal, ${ }^{24}$ L. Rebane,${ }^{24}$ A. Tiko, ${ }^{24}$ P. Eerola,${ }^{25}$ G. Fedi, ${ }^{25}$ M. Voutilainen, ${ }^{25}$ J. Härkönen, ${ }^{26}$ V. Karimäki, ${ }^{26}$ R. Kinnunen,${ }^{26}$ M. J. Kortelainen, ${ }^{26}$ T. Lampén, ${ }^{26}$ K. Lassila-Perini, ${ }^{26}$ S. Lehti, ${ }^{26}$ T. Lindén, ${ }^{26}$ P. Luukka ${ }^{26}$ T. Mäenpää, ${ }^{26}$ T. Peltola, ${ }^{26}$ E. Tuominen, ${ }^{26}$ J. Tuominiemi, ${ }^{26}$ E. Tuovinen,${ }^{26}$ L. Wendland, ${ }^{26}$ T. Tuuva, ${ }^{27}$ M. Besancon,${ }^{28}$ F. Couderc,${ }^{28}$ M. Dejardin, ${ }^{28}$ D. Denegri, ${ }^{28}$ B. Fabbro, ${ }^{28}$ J. L. Faure, ${ }^{28}$ F. Ferri, ${ }^{28}$ S. Ganjour, ${ }^{28}$ A. Givernaud, ${ }^{28}$ P. Gras,${ }^{28}$ G. Hamel de Monchenault,${ }^{28}$ P. Jarry, ${ }^{28}$ E. Locci, ${ }^{28}$ J. Malcles, ${ }^{28}$ A. Nayak, ${ }^{28}$ J. Rander, ${ }^{28}$ A. Rosowsky, ${ }^{28}$ M. Titov, ${ }^{28}$ S. Baffioni, ${ }^{29}$ F. Beaudette, ${ }^{29}$ P. Busson, ${ }^{29}$ C. Charlot, ${ }^{29}$ N. Daci, ${ }^{29}$ T. Dahms,${ }^{29}$ M. Dalchenko, ${ }^{29}$ L. Dobrzynski, ${ }^{29}$ A. Florent,${ }^{29}$

R. Granier de Cassagnac, ${ }^{29}$ M. Haguenauer, ${ }^{29}$ P. Miné, ${ }^{29}$ C. Mironov, ${ }^{29}$ I. N. Naranjo, ${ }^{29}$ M. Nguyen, ${ }^{29}$ C. Ochando, ${ }^{29}$ P. Paganini, ${ }^{29}$ D. Sabes,${ }^{29}$ R. Salerno, ${ }^{29}$ Y. Sirois, ${ }^{29}$ C. Veelken, ${ }^{29}$ Y. Yilmaz, ${ }^{29}$ A. Zabi, ${ }^{29}$ J.-L. Agram, ${ }^{30, p}$ J. Andrea, ${ }^{30}$ 
D. Bloch, ${ }^{30}$ J.-M. Brom,${ }^{30}$ E. C. Chabert, ${ }^{30}$ C. Collard, ${ }^{30}$ E. Conte, ${ }^{30, p}$ F. Drouhin, ${ }^{30, p}$ J.-C. Fontaine, ${ }^{30, p}$ D. Gelé,,${ }^{30}$ U. Goerlach,${ }^{30}$ C. Goetzmann, ${ }^{30}$ P. Juillot,${ }^{30}$ A.-C. Le Bihan, ${ }^{30}$ P. Van Hove,${ }^{30}$ S. Gadrat,${ }^{31}$ S. Beauceron,${ }^{32}$ N. Beaupere, ${ }^{32}$ G. Boudoul, ${ }^{32}$ S. Brochet, ${ }^{32}$ J. Chasserat,${ }^{32}$ R. Chierici, ${ }^{32}$ D. Contardo, ${ }^{32}$ P. Depasse, ${ }^{32}$ H. El Mamouni, ${ }^{32}$ J. Fan, ${ }^{32}$ J. Fay, ${ }^{32}$ S. Gascon, ${ }^{32}$ M. Gouzevitch, ${ }^{32}$ B. Ille, ${ }^{32}$ T. Kurca, ${ }^{32}$ M. Lethuillier, ${ }^{32}$ L. Mirabito, ${ }^{32}$ S. Perries, ${ }^{32}$ J. D. Ruiz Alvarez, ${ }^{32}$ L. Sgandurra, ${ }^{32}$ V. Sordini, ${ }^{32}$ M. Vander Donckt, ${ }^{32}$ P. Verdier, ${ }^{32}$ S. Viret, ${ }^{32}$ H. Xiao, ${ }^{32}$ Z. Tsamalaidze, ${ }^{33, q}$ C. Autermann ${ }^{34}$ S. Beranek ${ }^{34}$ M. Bontenackels,${ }^{34}$ B. Calpas,${ }^{34}$ M. Edelhoff, ${ }^{34}$ L. Feld, ${ }^{34}$ O. Hindrichs, ${ }^{34}$ K. Klein, ${ }^{34}$ A. Ostapchuk,${ }^{34}$ A. Perieanu, ${ }^{34}$ F. Raupach ${ }^{34}$ J. Sammet, ${ }^{34}$ S. Schael,${ }^{34}$ D. Sprenger, ${ }^{34}$ H. Weber, ${ }^{34}$ B. Wittmer, ${ }^{34}$ V. Zhukov, ${ }^{34, f}$ M. Ata,${ }^{35}$ J. Caudron, ${ }^{35}$ E. Dietz-Laursonn, ${ }^{35}$ D. Duchardt, ${ }^{35}$ M. Erdmann, ${ }^{35}$ R. Fischer, ${ }^{35}$ A. Güth, ${ }^{35}$ T. Hebbeker, ${ }^{35}$ C. Heidemann, ${ }^{35}$ K. Hoepfner, ${ }^{35}$ D. Klingebiel, ${ }^{35}$ S. Knutzen,${ }^{35}$ P. Kreuzer, ${ }^{35}$ M. Merschmeyer, ${ }^{35}$ A. Meyer, ${ }^{35}$ M. Olschewski, ${ }^{35}$ K. Padeken, ${ }^{35}$ P. Papacz, ${ }^{35}$ H. Reithler, ${ }^{35}$ S. A. Schmitz, ${ }^{35}$ L. Sonnenschein, ${ }^{35}$ D. Teyssier, ${ }^{35}$ S. Thüer, ${ }^{35}$ M. Weber, ${ }^{35}$ V. Cherepanov, ${ }^{36}$ Y. Erdogan,${ }^{36}$ G. Flügge, ${ }^{36}$ H. Geenen, ${ }^{36}$ M. Geisler, ${ }^{36}$ W. Haj Ahmad, ${ }^{36}$ F. Hoehle, ${ }^{36}$ B. Kargoll, ${ }^{36}$ T. Kress, ${ }^{36}$ Y. Kuessel, ${ }^{36}$ J. Lingemann, ${ }^{36, c}$ A. Nowack, ${ }^{36}$ I. M. Nugent ${ }^{36}$ L. Perchalla, ${ }^{36}$ O. Pooth ${ }^{36}$ A. Stahl, ${ }^{36}$ I. Asin, ${ }^{37}$ N. Bartosik, ${ }^{37}$ J. Behr, ${ }^{37}$ W. Behrenhoff, ${ }^{37}$ U. Behrens, ${ }^{37}$ A. J. Bell, ${ }^{37}$ M. Bergholz, ${ }^{37, r}$ A. Bethani, ${ }^{37}$ K. Borras, ${ }^{37}$ A. Burgmeier,${ }^{37}$ A. Cakir, ${ }^{37}$ L. Calligaris, ${ }^{37}$ A. Campbell, ${ }^{37}$ S. Choudhury, ${ }^{37}$ F. Costanza, ${ }^{37}$ C. Diez Pardos, ${ }^{37}$ S. Dooling, ${ }^{37}$ T. Dorland,${ }^{37}$ G. Eckerlin, ${ }^{37}$ D. Eckstein, ${ }^{37}$ T. Eichhorn, ${ }^{37}$ G. Flucke,${ }^{37}$ A. Geiser, ${ }^{37}$ A. Grebenyuk, ${ }^{37}$ P. Gunnellini, ${ }^{37}$ S. Habib,${ }^{37}$ J. Hauk,${ }^{37}$ G. Hellwig, ${ }^{37}$ M. Hempel,${ }^{37}$ D. Horton, ${ }^{37}$ H. Jung, ${ }^{37}$ M. Kasemann, ${ }^{37}$ P. Katsas, ${ }^{37}$ C. Kleinwort,${ }^{37}$ M. Krämer, ${ }^{37}$ D. Krücker, ${ }^{37}$ W. Lange, ${ }^{37}$ J. Leonard, ${ }^{37}$ K. Lipka ${ }^{37}$ W. Lohmann, ${ }^{37, \mathrm{r}}$ B. Lutz, ${ }^{37}$ R. Mankel, ${ }^{37}$ I. Marfin, ${ }^{37}$ I.-A. Melzer-Pellmann, ${ }^{37}$ A. B. Meyer,${ }^{37}$ J. Mnich, ${ }^{37}$ A. Mussgiller, ${ }^{37}$ S. Naumann-Emme, ${ }^{37}$ O. Novgorodova, ${ }^{37}$ F. Nowak,${ }^{37}$ H. Perrey, ${ }^{37}$ A. Petrukhin, ${ }^{37}$ D. Pitzl, ${ }^{37}$ R. Placakyte, ${ }^{37}$ A. Raspereza, ${ }^{37}$ P. M. Ribeiro Cipriano, ${ }^{37}$ C. Riedl,,${ }^{37}$ E. Ron, ${ }^{37}$ M. Ö. Sahin,,${ }^{37}$ J. Salfeld-Nebgen, ${ }^{37}$ R. Schmidt, ${ }^{37, \mathrm{r}}$ T. Schoerner-Sadenius, ${ }^{37}$ M. Schröder, ${ }^{37}$ M. Stein, ${ }^{37}$ A. D. R. Vargas Trevino, ${ }^{37}$ R. Walsh,${ }^{37}$ C. Wissing,${ }^{37}$ M. Aldaya Martin, ${ }^{38}$ V. Blobel,${ }^{38}$ H. Enderle, ${ }^{38}$ J. Erfle, ${ }^{38}$ E. Garutti, ${ }^{38}$ M. Görner, ${ }^{38}$ M. Gosselink,${ }^{38}$ J. Haller, ${ }^{38}$ K. Heine, ${ }^{38}$ R. S. Höing, ${ }^{38}$ H. Kirschenmann, ${ }^{38}$ R. Klanner, ${ }^{38}$ R. Kogler, ${ }^{38}$ J. Lange, ${ }^{38}$ I. Marchesini ${ }^{38}$ J. Ott, ${ }^{38}$ T. Peiffer,${ }^{38}$ N. Pietsch,${ }^{38}$ D. Rathjens, ${ }^{38}$ C. Sander, ${ }^{38}$ H. Schettler, ${ }^{38}$ P. Schleper, ${ }^{38}$ E. Schlieckau, ${ }^{38}$ A. Schmidt, ${ }^{38}$ M. Seidel,${ }^{38}$ J. Sibille, ${ }^{38, s}$ V. Sola,${ }^{38}$ H. Stadie, ${ }^{38}$ G. Steinbrück, ${ }^{38}$ D. Troendle, ${ }^{38}$ E. Usai, ${ }^{38}$ L. Vanelderen, ${ }^{38}$ C. Barth, ${ }^{39}$ C. Baus, ${ }^{39}$ J. Berger, ${ }^{39}$ C. Böser, ${ }^{39}$ E. Butz, ${ }^{39}$ T. Chwalek, ${ }^{39}$ W. De Boer, ${ }^{39}$ A. Descroix, ${ }^{39}$ A. Dierlamm, ${ }^{39}$ M. Feindt, ${ }^{39}$ M. Guthoff, ${ }^{39, c}$ F. Hartmann, ${ }^{39, c}$ T. Hauth, ${ }^{39, \mathrm{c}}$ H. Held, ${ }^{39}$ K. H. Hoffmann, ${ }^{39}$ U. Husemann, ${ }^{39}$ I. Katkov, ${ }^{39, f}$ A. Kornmayer, ${ }^{39, c}$ E. Kuznetsova, ${ }^{39}$ P. Lobelle Pardo ${ }^{39}$ D. Martschei, ${ }^{39}$ M. U. Mozer, ${ }^{39}$ Th. Müller, ${ }^{39}$ M. Niegel,${ }^{39}$ A. Nürnberg, ${ }^{39}$ O. Oberst, ${ }^{39}$ G. Quast,${ }^{39}$ K. Rabbertz, ${ }^{39}$ F. Ratnikov, ${ }^{39}$ S. Röcker ${ }^{39}$ F.-P. Schilling, ${ }^{39}$ G. Schott, ${ }^{39}$ H. J. Simonis,${ }^{39}$ F. M. Stober, ${ }^{39}$ R. Ulrich, ${ }^{39}$ J. Wagner-Kuhr, ${ }^{39}$ S. Wayand, ${ }^{39}$ T. Weiler, ${ }^{39}$ R. Wolf, ${ }^{39}$ M. Zeise, ${ }^{39}$ G. Anagnostou, ${ }^{40}$ G. Daskalakis,${ }^{40}$ T. Geralis, ${ }^{40}$ S. Kesisoglou, ${ }^{40}$ A. Kyriakis, ${ }^{40}$ D. Loukas, ${ }^{40}$ A. Markou, ${ }^{40}$ C. Markou, ${ }^{40}$ E. Ntomari ${ }^{40}$ I. Topsis-giotis, ${ }^{40}$ L. Gouskos, ${ }^{41}$ A. Panagiotou, ${ }^{41}$ N. Saoulidou, ${ }^{41}$ E. Stiliaris, ${ }^{41}$ X. Aslanoglou, ${ }^{42}$ I. Evangelou, ${ }^{42}$ G. Flouris, ${ }^{42}$ C. Foudas, ${ }^{42}$ P. Kokkas, ${ }^{42}$ N. Manthos, ${ }^{42}$ I. Papadopoulos,${ }^{42}$ E. Paradas, ${ }^{42}$ G. Bencze, ${ }^{43}$ C. Hajdu, ${ }^{43}$ P. Hidas, ${ }^{43}$ D. Horvath, ${ }^{43, t}$ F. Sikler,${ }^{43}$

V. Veszpremi, ${ }^{43}$ G. Vesztergombi, ${ }^{43, u}$ A. J. Zsigmond, ${ }^{43}$ N. Beni, ${ }^{44}$ S. Czellar, ${ }^{44}$ J. Molnar, ${ }^{44}$ J. Palinkas, ${ }^{44}$ Z. Szillasi, ${ }^{44}$ J. Karancsi, ${ }^{45}$ P. Raics, ${ }^{45}$ Z. L. Trocsanyi,${ }^{45}$ B. Ujvari, ${ }^{45}$ S. K. Swain, ${ }^{46, v}$ S. B. Beri, ${ }^{47}$ V. Bhatnagar, ${ }^{47}$ N. Dhingra, ${ }^{47}$ R. Gupta, ${ }^{47}$ M. Kaur, ${ }^{47}$ M. Z. Mehta, ${ }^{47}$ M. Mittal,${ }^{47}$ N. Nishu, ${ }^{47}$ A. Sharma,${ }^{47}$ J. B. Singh, ${ }^{47}$ Ashok Kumar ${ }^{48}$ Arun Kumar ${ }^{48}$ S. Ahuja, ${ }^{48}$ A. Bhardwaj, ${ }^{48}$ B. C. Choudhary, ${ }^{48}$ A. Kumar, ${ }^{48}$ S. Malhotra ${ }^{48}$ M. Naimuddin, ${ }^{48}$ K. Ranjan, ${ }^{48}$ P. Saxena, ${ }^{48}$ V. Sharma, ${ }^{48}$ R. K. Shivpuri, ${ }^{48}$ S. Banerjee, ${ }^{49}$ S. Bhattacharya, ${ }^{49}$ K. Chatterjee, ${ }^{49}$ S. Dutta, ${ }^{49}$ B. Gomber ${ }^{49}$ Sa. Jain, ${ }^{49}$ Sh. Jain, ${ }^{49}$ R. Khurana, ${ }^{49}$ A. Modak, ${ }^{49}$ S. Mukherjee, ${ }^{49}$ D. Roy, ${ }^{49}$ S. Sarkar,${ }^{49}$ M. Sharan ${ }^{49}$ A. P. Singh,${ }^{49}$ A. Abdulsalam, ${ }^{50}$ D. Dutta, ${ }^{50}$ S. Kailas, ${ }^{50}$ V. Kumar, ${ }^{50}$ A. K. Mohanty,${ }^{50, c}$ L. M. Pant, ${ }^{50}$ P. Shukla, ${ }^{50}$ A. Topkar, ${ }^{50}$ T. Aziz, ${ }^{51}$ R. M. Chatterjee,${ }^{51}$

S. Ganguly ${ }^{51}$ S. Ghosh, ${ }^{51}$ M. Guchait, ${ }^{51, w}$ A. Gurtu, ${ }^{51, x}$ G. Kole, ${ }^{51}$ S. Kumar, ${ }^{51}$ M. Maity, ${ }^{51, y}$ G. Majumder, ${ }^{51}$

K. Mazumdar, ${ }^{51}$ G. B. Mohanty, ${ }^{51}$ B. Parida, ${ }^{51}$ K. Sudhakar, ${ }^{51}$ N. Wickramage, ${ }^{51, z}$ S. Banerjee ${ }^{52}$ S. Dugad, ${ }^{52}$ H. Arfaei, ${ }^{53}$ H. Bakhshiansohi, ${ }^{53}$ H. Behnamian, ${ }^{53}$ S. M. Etesami, ${ }^{53 \text { aa }}$ A. Fahim, ${ }^{53, \text { bb }}$ A. Jafari, ${ }^{53}$ M. Khakzad, ${ }^{53}$

M. Mohammadi Najafabadi, ${ }^{53}$ M. Naseri, ${ }^{53}$ S. Paktinat Mehdiabadi, ${ }^{53}$ B. Safarzadeh, ${ }^{53, c c}$ M. Zeinali, ${ }^{53}$ M. Grunewald, ${ }^{54}$ M. Abbrescia ${ }^{55 a, 55 b}$ L. Barbone,${ }^{55 a, 55 b}$ C. Calabria, ${ }^{55 a, 55 b}$ S. S. Chhibra, ${ }^{55 a, 55 b}$ A. Colaleo, ${ }^{55 a}$ D. Creanza, ${ }^{55,55 c}$ N. De Filippis, ${ }^{55 a, 55 c}$ M. De Palma,${ }^{55 a, 55 b}$ L. Fiore, ${ }^{55 a}$ G. Iaselli, ${ }^{55 a, 55 c}$ G. Maggi, ${ }^{55 a, 55 c}$ M. Maggi, ${ }^{55 a}$ B. Marangelli, ${ }^{55 a, 55 b}$ S. My ${ }^{55 a, 55 c}$ S. Nuzzo, ${ }^{55 a, 55 b}$ N. Pacifico, ${ }^{55 a}$ A. Pompili, ${ }^{55,55 b}$ G. Pugliese, ${ }^{55 a, 55 c}$ R. Radogna, ${ }^{55 a, 55 b}$ G. Selvaggi, ${ }^{55 a, 55 b}$ L. Silvestris, ${ }^{55 \mathrm{a}}$ G. Singh, ${ }^{55,55 \mathrm{~b}}$ R. Venditti, ${ }^{55 \mathrm{a}, 55 \mathrm{~b}}$ P. Verwilligen, ${ }^{55 \mathrm{a}}$ G. Zito, ${ }^{55 \mathrm{a}}$ G. Abbiendi, ${ }^{56 \mathrm{a}}$ A. C. Benvenuti, ${ }^{56 \mathrm{a}}$

D. Bonacorsi, ${ }^{56 \mathrm{a}, 56 \mathrm{~b}}$ S. Braibant-Giacomelli, ${ }^{56 \mathrm{a}, 56 \mathrm{~b}}$ L. Brigliadori, ${ }^{56,56 \mathrm{~b}}$ R. Campanini, ${ }^{56,56 \mathrm{~b}}$ P. Capiluppi, ${ }^{56 \mathrm{a}, 56 \mathrm{~b}}$

A. Castro, ${ }^{56 a, 56 b}$ F. R. Cavallo, ${ }^{56 a}$ G. Codispoti, ${ }^{56,56 b}$ M. Cuffiani, ${ }^{56 a, 56 b}$ G. M. Dallavalle, ${ }^{56 a}$ F. Fabbri, ${ }^{56 a}$ A. Fanfani, ${ }^{56 a, 56 b}$ 
D. Fasanella, ${ }^{56,56 \mathrm{~b}}$ P. Giacomelli, ${ }^{56 \mathrm{a}}$ C. Grandi, ${ }^{56 \mathrm{a}}$ L. Guiducci, ${ }^{56,56 \mathrm{~b}}$ S. Marcellini, ${ }^{56 \mathrm{a}}$ G. Masetti, ${ }^{56 \mathrm{a}}$ M. Meneghelli, ${ }^{56 \mathrm{a}, 56 \mathrm{~b}}$

A. Montanari, ${ }^{56 \mathrm{a}}$ F. L. Navarria, ${ }^{56 \mathrm{a}, 56 \mathrm{~b}}$ F. Odorici, ${ }^{56 \mathrm{a}}$ A. Perrotta, ${ }^{56 \mathrm{a}}$ F. Primavera, ${ }^{56,56 \mathrm{~b}}$ A. M. Rossi, ${ }^{56 a, 56 \mathrm{~b}}$ T. Rovelli, ${ }^{56 a, 56 \mathrm{~b}}$ G. P. Siroli, ${ }^{56,56 b}$ N. Tosi, ${ }^{56 a, 56 b}$ R. Travaglini, ${ }^{56,56 b}$ S. Albergo, ${ }^{57 a, 57 b}$ G. Cappello, ${ }^{57 a}$ M. Chiorboli, ${ }^{57 a, 57 b}$ S. Costa, ${ }^{57 a, 57 b}$ F. Giordano, ${ }^{57 a, c}$ R. Potenza, ${ }^{57,57 b}$ A. Tricomi,${ }^{57 a, 57 b}$ C. Tuve ${ }^{57 a, 57 b}$ G. Barbagli, ${ }^{58 a}$ V. Ciulli, ${ }^{58 a, 58 b}$ C. Civinini, ${ }^{58 a}$ R. D’Alessandro, ${ }^{58 a, 58 b}$ E. Focardi, ${ }^{58 a, 58 b}$ E. Gallo, ${ }^{58 a}$ S. Gonzi, ${ }^{58 a, 58 b}$ V. Gori, ${ }^{58 a, 58 b}$ P. Lenzi, ${ }^{58 a, 58 b}$ M. Meschini, ${ }^{58 a}$ S. Paoletti, ${ }^{58 \mathrm{a}}$ G. Sguazzoni, ${ }^{58 \mathrm{a}}$ A. Tropiano, ${ }^{58 \mathrm{a}, 58 \mathrm{~b}}$ L. Benussi, ${ }^{59}$ S. Bianco, ${ }^{59}$ F. Fabbri, ${ }^{59}$ D. Piccolo,${ }^{59}$ P. Fabbricatore, ${ }^{60 \mathrm{a}}$ R. Ferretti, ${ }^{60 \mathrm{a}, 60 \mathrm{~b}}$ F. Ferro, ${ }^{60 \mathrm{a}}$ M. Lo Vetere, ${ }^{60 \mathrm{a}, 60 \mathrm{~b}}$ R. Musenich, ${ }^{60 \mathrm{a}}$ E. Robutti, ${ }^{60 \mathrm{a}} \mathrm{S}$. Tosi, ${ }^{60 \mathrm{a}, 60 \mathrm{~b}}$ A. Benaglia, ${ }^{61 \mathrm{a}}$ M. E. Dinardo, ${ }^{61 a, 61 b}$ S. Fiorendi, ${ }^{61 a, 61 b, c}$ S. Gennai, ${ }^{61 a}$ A. Ghezzi, ${ }^{61 a, 61 b}$ P. Govoni, ${ }^{61 a, 61 b}$ M. T. Lucchini, ${ }^{61 a, 61 b, c}$ S. Malvezzi, ${ }^{61 \mathrm{a}}$ R. A. Manzoni, ${ }^{61 \mathrm{a}, 61 \mathrm{~b}, \mathrm{c}}$ A. Martelli, ${ }^{61 \mathrm{a}, 61 \mathrm{~b}, \mathrm{c}}$ D. Menasce, ${ }^{61 \mathrm{a}}$ L. Moroni, ${ }^{61 \mathrm{a}}$ M. Paganoni, ${ }^{61 \mathrm{a}, 61 \mathrm{~b}}$ D. Pedrini, ${ }^{61 \mathrm{a}}$ S. Ragazzi, ${ }^{61 \mathrm{a}, 61 \mathrm{~b}}$ N. Redaelli, ${ }^{61 \mathrm{a}}$ T. Tabarelli de Fatis, ${ }^{61,61 \mathrm{~b}}$ S. Buontempo ${ }^{62 \mathrm{a}}$ N. Cavallo, ${ }^{62 a, 62 \mathrm{c}}$ F. Fabozzi, ${ }^{62 \mathrm{a}, 62 \mathrm{c}}$

A. O. M. Iorio, ${ }^{62 a, 62 b}$ L. Lista, ${ }^{62 a}$ S. Meola,${ }^{62 a, 62 d, c}$ M. Merola, ${ }^{62 a}$ P. Paolucci, ${ }^{62 a, c}$ P. Azzi, ${ }^{63 a}$ N. Bacchetta, ${ }^{63 a}$ D. Bisello,${ }^{63 a, 63 b}$ A. Branca, ${ }^{63 a, 63 b}$ R. Carlin, ${ }^{63 a, 63 b}$ P. Checchia, ${ }^{63 a}$ T. Dorigo ${ }^{63 a}$ U. Dosselli, ${ }^{63 a}$ M. Galanti, ${ }^{63 a, 63 b, c}$ F. Gasparini, ${ }^{63 a, 63 b}$ U. Gasparini, ${ }^{63 a, 63 b}$ P. Giubilato, ${ }^{63 a, 63 b}$ A. Gozzelino, ${ }^{63 a}$ K. Kanishchev, ${ }^{63 a, 63 c}$ S. Lacaprara, ${ }^{63 a}$ I. Lazzizzera, ${ }^{63 a, 63 c}$ M. Margoni, ${ }^{63 a, 63 b}$ A. T. Meneguzzo, ${ }^{63 a, 63 b}$ M. Nespolo, ${ }^{63 a}$ M. Passaseo, ${ }^{63 a}$ J. Pazzini, ${ }^{63 a, 63 b}$ M. Pegoraro, ${ }^{63 a}$ N. Pozzobon, ${ }^{63 a, 63 b}$ P. Ronchese, ${ }^{63 a, 63 b}$ F. Simonetto, ${ }^{63 a, 63 b}$ E. Torassa, ${ }^{63 a}$ M. Tosi, ${ }^{63 a, 63 b}$ P. Zotto, ${ }^{63 a, 63 b}$ A. Zucchetta, ${ }^{63 a, 63 b}$ G. Zumerle, ${ }^{63 a, 63 b}$ M. Gabusi, ${ }^{64 a, 64 b}$ S. P. Ratti, ${ }^{64 a, 64 b}$ C. Riccardi, ${ }^{64 a, 64 b}$ P. Vitulo, ${ }^{64 a, 64 b}$ M. Biasini, ${ }^{65 a, 65 b}$ G. M. Bilei, ${ }^{65 a}$ L. Fanò ${ }^{65 a, 65 b}$ P. Lariccia, ${ }^{65 a, 65 b}$ G. Mantovani, ${ }^{65 a, 65 b}$ M. Menichelli, ${ }^{65 a}$ A. Nappi,${ }^{65 a, 65 b, a}$ F. Romeo, ${ }^{65 a, 65 b}$ A. Saha ${ }^{65 a}$ A. Santocchia, ${ }^{65 a, 65 b}$ A. Spiezia, ${ }^{65 a, 65 b}$ K. Androsov, ${ }^{66 a, d d}$ P. Azzurri, ${ }^{66 a}$ G. Bagliesi ${ }^{66 a}$ J. Bernardini, ${ }^{66 a}$ T. Boccali ${ }^{66 a}$ G. Broccolo, ${ }^{66 a, 66 c}$ R. Castaldi, ${ }^{66 a}$ M. A. Ciocci, ${ }^{66 a, d d}$ R. Dell’Orso, ${ }^{66 a}$ F. Fiori, ${ }^{66 a, 66 c}$ L. Foà, ${ }^{66 a, 66 c}$ A. Giassi, ${ }^{66 a}$ M. T. Grippo, ${ }^{66 a, d d}$ A. Kraan, ${ }^{66 a}$ F. Ligabue, ${ }^{66 a, 66 c}$ T. Lomtadze, ${ }^{66 a}$ L. Martini, ${ }^{66 a, 66 b}$ A. Messineo, ${ }^{66 a, 66 b}$ C. S. Moon, ${ }^{66 a, e e}$ F. Palla, ${ }^{66 a}$ A. Rizzi, ${ }^{66 a, 66 b}$ A. Savoy-Navarro, ${ }^{66, f f}$ A. T. Serban, ${ }^{66 a}$ P. Spagnolo ${ }^{66 a}$ P. Squillacioti ${ }^{66 a, d d}$ R. Tenchini, ${ }^{66 a}$ G. Tonelli, ${ }^{66 a, 66 b}$ A. Venturi, ${ }^{66 a}$ P. G. Verdini, ${ }^{66 a}$ C. Vernieri, ${ }^{66 a, 66 c}$ L. Barone ${ }^{67 a, 67 b}$ F. Cavallari, ${ }^{67 a}$ D. Del Re, ${ }^{67 a, 67 b}$ M. Diemoz, ${ }^{67 a}$ M. Grassi, ${ }^{67 a, 67 b}$ C. Jorda, ${ }^{67 a}$ E. Longo, ${ }^{67 a, 67 b}$ F. Margaroli ${ }^{67 a, 67 b}$ P. Meridiani, ${ }^{67 a}$ F. Micheli, ${ }^{67 a, 67 b}$ S. Nourbakhsh,${ }^{67 a, 67 b}$ G. Organtini, ${ }^{67 a, 67 b}$ R. Paramatti, ${ }^{67 a}$ S. Rahatlou, ${ }^{67 a, 67 b}$ C. Rovelli, ${ }^{67 a}$ L. Soffi, ${ }^{67 a, 67 b}$ P. Traczyk, ${ }^{67 a, 67 b}$ N. Amapane, ${ }^{68 a, 68 b}$ R. Arcidiacono, ${ }^{68 a, 68 c}$ S. Argiro, ${ }^{68 a, 68 b}$ M. Arneodo, ${ }^{68 a, 68 c}$ R. Bellan, ${ }^{68 a, 68 b}$ C. Biino, ${ }^{68 a}$ N. Cartiglia, ${ }^{68 a}$ S. Casasso, ${ }^{68,68 b}$ M. Costa ${ }^{68 a, 68 b}$ A. Degano, ${ }^{68,68 b}$ N. Demaria, ${ }^{68 a}$ C. Mariotti, ${ }^{68 a}$ S. Maselli, ${ }^{68 a}$ E. Migliore, ${ }^{68 a, 68 b}$ V. Monaco ${ }^{68 \mathrm{a}, 68 \mathrm{~b}}$ M. Musich, ${ }^{68 \mathrm{a}}$ M. M. Obertino, ${ }^{68 \mathrm{a}, 68 \mathrm{c}}$ G. Ortona,${ }^{68 \mathrm{a}, 68 \mathrm{~b}}$ L. Pacher,${ }^{68 \mathrm{a}, 68 \mathrm{~b}}$ N. Pastrone, ${ }^{68 \mathrm{a}}$ M. Pelliccioni, ${ }^{68 \mathrm{a}, \mathrm{c}}$ A. Potenza, ${ }^{68 \mathrm{a}, 68 \mathrm{~b}}$ A. Romero, ${ }^{68 \mathrm{a}, 68 \mathrm{~b}}$ M. Ruspa ${ }^{68 \mathrm{a}, 68 \mathrm{c}}$ R. Sacchi, ${ }^{68,68 \mathrm{~b}}$ A. Solano, ${ }^{68 \mathrm{a}, 68 \mathrm{~b}}$ A. Staiano, ${ }^{68 \mathrm{a}}$ U. Tamponi, ${ }^{68 \mathrm{a}}$ S. Belforte,${ }^{69 a}$ V. Candelise,${ }^{69 a, 69 b}$ M. Casarsa, ${ }^{69 a}$ F. Cossutti, ${ }^{69 a, c}$ G. Della Ricca, ${ }^{69 a, 69 b}$ B. Gobbo, ${ }^{69 a}$ C. La Licata, ${ }^{69 a, 69 b}$ M. Marone ${ }^{69 a, 69 b}$ D. Montanino, ${ }^{69 a, 69 b}$ A. Penzo, ${ }^{69 a}$ A. Schizzi, ${ }^{69 a, 69 b}$ T. Umer, ${ }^{69 a, 69 b}$ A. Zanetti, ${ }^{69 a}$ S. Chang, ${ }^{70}$ T. Y. Kim, ${ }^{70}$ S. K. Nam, ${ }^{70}$ D. H. Kim, ${ }^{71}$ G. N. Kim,${ }^{71}$ J. E. Kim, ${ }^{71}$ D. J. Kong, ${ }^{71}$ S. Lee, ${ }^{71}$ Y. D. Oh,${ }^{71}$ H. Park, ${ }^{71}$ D. C. Son, ${ }^{71}$ J. Y. Kim, ${ }^{72}$ Zero J. Kim, ${ }^{72}$ S. Song, ${ }^{72}$ S. Choi, ${ }^{73}$ D. Gyun, ${ }^{73}$ B. Hong, ${ }^{73}$ M. Jo, ${ }^{73}$ H. Kim,${ }^{73}$ Y. Kim, ${ }^{73}$ K. S. Lee, ${ }^{73}$ S. K. Park, ${ }^{73}$ Y. Roh,${ }^{73}$ M. Choi ${ }^{74}$ J. H. Kim,${ }^{74}$ C. Park, ${ }^{74}$ I. C. Park, ${ }^{74}$ S. Park, ${ }^{74}$ G. Ryu, ${ }^{74}$ Y. Choi,${ }^{75}$ Y. K. Choi, ${ }^{75}$ J. Goh,${ }^{75}$ M. S. Kim, ${ }^{75}$ E. Kwon, ${ }^{75}$ B. Lee, ${ }^{75}$ J. Lee, ${ }^{75}$ S. Lee,${ }^{75}$ H. Seo, ${ }^{75}$ I. Yu ${ }^{75}$ A. Juodagalvis, ${ }^{76}$ H. Castilla-Valdez, ${ }^{77}$ E. De La Cruz-Burelo, ${ }^{77}$ I. Heredia-de La Cruz, ${ }^{77, g g}$ R. Lopez-Fernandez, ${ }^{77}$ J. Martínez-Ortega, ${ }^{77}$ A. Sanchez-Hernandez, ${ }^{77}$

L. M. Villasenor-Cendejas, ${ }^{77}$ S. Carrillo Moreno, ${ }^{78}$ F. Vazquez Valencia ${ }^{78}$ H. A. Salazar Ibarguen, ${ }^{79}$ E. Casimiro Linares ${ }^{80}$ A. Morelos Pineda, ${ }^{80}$ D. Krofcheck, ${ }^{81}$ P. H. Butler, ${ }^{82}$ R. Doesburg, ${ }^{82}$ S. Reucroft, ${ }^{82}$ H. Silverwood, ${ }^{82}$ M. Ahmad, ${ }^{83}$ M. I. Asghar, ${ }^{83}$ J. Butt, ${ }^{83}$ H. R. Hoorani, ${ }^{83}$ S. Khalid ${ }^{83}$ W. A. Khan, ${ }^{83}$ T. Khurshid, ${ }^{83}$ S. Qazi, ${ }^{83}$ M. A. Shah, ${ }^{83}$ M. Shoaib, ${ }^{83}$ H. Bialkowska ${ }^{84}$ M. Bluj, ${ }^{84, h h}$ B. Boimska,${ }^{84}$ T. Frueboes,${ }^{84}$ M. Górski, ${ }^{84}$ M. Kazana, ${ }^{84}$ K. Nawrocki,${ }^{84}$

K. Romanowska-Rybinska, ${ }^{84}$ M. Szleper,${ }^{84}$ G. Wrochna ${ }^{84}$ P. Zalewski, ${ }^{84}$ G. Brona, ${ }^{85}$ K. Bunkowski, ${ }^{85}$ M. Cwiok, ${ }^{85}$ W. Dominik, ${ }^{85}$ K. Doroba ${ }^{85}$ A. Kalinowski, ${ }^{85}$ M. Konecki, ${ }^{85}$ J. Krolikowski, ${ }^{85}$ M. Misiura, ${ }^{85}$ W. Wolszczak,${ }^{85}$ P. Bargassa, ${ }^{86}$ C. Beirão Da Cruz E Silva, ${ }^{86}$ P. Faccioli,${ }^{86}$ P. G. Ferreira Parracho, ${ }^{86}$ M. Gallinaro ${ }^{86}$ F. Nguyen, ${ }^{86}$ J. Rodrigues Antunes, ${ }^{86}$ J. Seixas, ${ }^{86, \mathrm{C}}$ J. Varela, ${ }^{86}$ P. Vischia, ${ }^{86}$ S. Afanasiev, ${ }^{87}$ P. Bunin, ${ }^{87}$ I. Golutvin, ${ }^{87}$ I. Gorbunov, ${ }^{87}$ V. Karjavin, ${ }^{87}$

V. Konoplyanikov, ${ }^{87}$ G. Kozlov, ${ }^{87}$ A. Lanev ${ }^{87}$ A. Malakhov, ${ }^{87}$ V. Matveev, ${ }^{87}$ P. Moisenz,${ }^{87}$ V. Palichik,${ }^{87}$ V. Perelygin, ${ }^{87}$ M. Savina, ${ }^{87}$ S. Shmatov, ${ }^{87}$ N. Skatchkov, ${ }^{87}$ V. Smirnov, ${ }^{87}$ A. Zarubin,${ }^{87}$ V. Golovtsov, ${ }^{88}$ Y. Ivanov, ${ }^{88}$ V. Kim, ${ }^{88}$ P. Levchenko, ${ }^{88}$ V. Murzin, ${ }^{88}$ V. Oreshkin, ${ }^{88}$ I. Smirnov, ${ }^{88}$ V. Sulimov, ${ }^{88}$ L. Uvarov, ${ }^{88}$ S. Vavilov, ${ }^{88}$ A. Vorobyev ${ }^{88}$ An. Vorobyev, ${ }^{88}$ Yu. Andreev, ${ }^{89}$ A. Dermenev, ${ }^{89}$ S. Gninenko, ${ }^{89}$ N. Golubev, ${ }^{89}$ M. Kirsanov, ${ }^{89}$ N. Krasnikov, ${ }^{89}$ A. Pashenkov, ${ }^{89}$ D. Tlisov, ${ }^{89}$ A. Toropin, ${ }^{89}$ V. Epshteyn, ${ }^{90}$ V. Gavrilov,${ }^{90}$ N. Lychkovskaya, ${ }^{90}$ V. Popov,${ }^{90}$ G. Safronov, ${ }^{90}$ S. Semenov, ${ }^{90}$ A. Spiridonov, ${ }^{90}$ V. Stolin, ${ }^{90}$ E. Vlasov, ${ }^{90}$ A. Zhokin, ${ }^{90}$ V. Andreev, ${ }^{91}$ M. Azarkin, ${ }^{91}$ I. Dremin, ${ }^{91}$ 
M. Kirakosyan, ${ }^{91}$ A. Leonidov, ${ }^{91}$ G. Mesyats, ${ }^{91}$ S. V. Rusakov, ${ }^{91}$ A. Vinogradov, ${ }^{91}$ A. Belyaev, ${ }^{92}$ E. Boos,${ }^{92}$ V. Bunichev,${ }^{92}$ M. Dubinin, ${ }^{92, \mathrm{~h}}$ L. Dudko, ${ }^{92}$ A. Ershov, ${ }^{92}$ V. Klyukhin, ${ }^{92}$ O. Kodolova, ${ }^{92}$ I. Lokhtin, ${ }^{92}$ A. Markina, ${ }^{92}$ S. Obraztsov, ${ }^{92}$ S. Petrushanko, ${ }^{92}$ V. Savrin, ${ }^{92}$ A. Snigirev, ${ }^{92}$ I. Azhgirey, ${ }^{93}$ I. Bayshev, ${ }^{93}$ S. Bitioukov, ${ }^{93}$ V. Kachanov, ${ }^{93}$ A. Kalinin, ${ }^{93}$ D. Konstantinov, ${ }^{93}$ V. Krychkine, ${ }^{93}$ V. Petrov, ${ }^{93}$ R. Ryutin, ${ }^{93}$ A. Sobol,${ }^{93}$ L. Tourtchanovitch, ${ }^{93}$ S. Troshin, ${ }^{93}$ N. Tyurin,${ }^{93}$ A. Uzunian, ${ }^{93}$ A. Volkov, ${ }^{93}$ P. Adzic, ${ }^{94, i i}$ M. Djordjevic, ${ }^{94}$ M. Ekmedzic,${ }^{94}$ J. Milosevic, ${ }^{94}$ M. Aguilar-Benitez, ${ }^{95}$ J. Alcaraz Maestre, ${ }^{95}$ C. Battilana,${ }^{95}$ E. Calvo, ${ }^{95}$ M. Cerrada ${ }^{95}$ M. Chamizo Llatas, ${ }^{95, c}$ N. Colino, ${ }^{95}$ B. De La Cruz,${ }^{95}$ A. Delgado Peris,${ }^{95}$ D. Domínguez Vázquez,${ }^{95}$ C. Fernandez Bedoya,${ }^{95}$ J. P. Fernández Ramos, ${ }^{95}$ A. Ferrando,${ }^{95}$ J. Flix,${ }^{95}$ M. C. Fouz,${ }^{95}$ P. Garcia-Abia, ${ }^{95}$ O. Gonzalez Lopez,${ }^{95}$ S. Goy Lopez,${ }^{95}$ J. M. Hernandez,${ }^{95}$ M. I. Josa, ${ }^{95}$ G. Merino, ${ }^{95}$

E. Navarro De Martino, ${ }^{95}$ J. Puerta Pelayo, ${ }^{95}$ A. Quintario Olmeda, ${ }^{95}$ I. Redondo, ${ }^{95}$ L. Romero, ${ }^{95}$ M. S. Soares, ${ }^{95}$

C. Willmott, ${ }^{95}$ C. Albajar, ${ }^{96}$ J. F. de Trocóniz, ${ }^{96}$ H. Brun, ${ }^{97}$ J. Cuevas, ${ }^{97}$ J. Fernandez Menendez, ${ }^{97}$ S. Folgueras, ${ }^{97}$

I. Gonzalez Caballero, ${ }^{97}$ L. Lloret Iglesias, ${ }^{97}$ J. A. Brochero Cifuentes, ${ }^{98}$ I. J. Cabrillo, ${ }^{98}$ A. Calderon, ${ }^{98}$ S. H. Chuang, ${ }^{98}$

J. Duarte Campderros, ${ }^{98}$ M. Fernandez,${ }^{98}$ G. Gomez, ${ }^{98}$ J. Gonzalez Sanchez, ${ }^{98}$ A. Graziano, ${ }^{98}$ A. Lopez Virto, ${ }^{98}$ J. Marco, ${ }^{98}$ R. Marco,${ }^{98}$ C. Martinez Rivero, ${ }^{98}$ F. Matorras, ${ }^{98}$ F. J. Munoz Sanchez,${ }^{98}$ J. Piedra Gomez, ${ }^{98}$ T. Rodrigo, ${ }^{98}$

A. Y. Rodríguez-Marrero, ${ }^{98}$ A. Ruiz-Jimeno,${ }^{98}$ L. Scodellaro, ${ }^{98}$ I. Vila,${ }^{98}$ R. Vilar Cortabitarte, ${ }^{98}$ D. Abbaneo, ${ }^{99}$ E. Auffray, ${ }^{99}$ G. Auzinger, ${ }^{99}$ M. Bachtis, ${ }^{99}$ P. Baillon, ${ }^{99}$ A. H. Ball, ${ }^{99}$ D. Barney, ${ }^{99}$ J. Bendavid, ${ }^{99}$ L. Benhabib, ${ }^{99}$ J. F. Benitez, ${ }^{99}$

C. Bernet, ${ }^{99, i}$ G. Bianchi, ${ }^{99}$ P. Bloch ${ }^{99}$ A. Bocci,${ }^{99}$ A. Bonato, ${ }^{99}$ O. Bondu, ${ }^{99}$ C. Botta, ${ }^{99}$ H. Breuker,${ }^{99}$ T. Camporesi,${ }^{99}$ G. Cerminara, ${ }^{99}$ T. Christiansen, ${ }^{99}$ J. A. Coarasa Perez, ${ }^{99}$ S. Colafranceschi, ${ }^{99, j j}$ M. D'Alfonso, ${ }^{99}$ D. d'Enterria, ${ }^{99}$

A. Dabrowski, ${ }^{99}$ A. David, ${ }^{99}$ F. De Guio, ${ }^{99}$ A. De Roeck, ${ }^{99}$ S. De Visscher, ${ }^{99}$ S. Di Guida,${ }^{99}$ M. Dobson, ${ }^{99}$

N. Dupont-Sagorin, ${ }^{99}$ A. Elliott-Peisert, ${ }^{99}$ J. Eugster, ${ }^{99}$ G. Franzoni, ${ }^{99}$ W. Funk, ${ }^{99}$ M. Giffels, ${ }^{99}$ D. Gigi,${ }^{99}$ K. Gill, ${ }^{99}$ M. Girone, ${ }^{99}$ M. Giunta, ${ }^{99}$ F. Glege, ${ }^{99}$ R. Gomez-Reino Garrido, ${ }^{99}$ S. Gowdy, ${ }^{99}$ R. Guida,${ }^{99}$ J. Hammer,${ }^{99}$ M. Hansen, ${ }^{99}$ P. Harris, ${ }^{99}$ A. Hinzmann, ${ }^{99}$ V. Innocente, ${ }^{99}$ P. Janot, ${ }^{99}$ E. Karavakis, ${ }^{99}$ K. Kousouris, ${ }^{99}$ K. Krajczar, ${ }^{99}$ P. Lecoq, ${ }^{99}$ C. Lourenço, ${ }^{99}$ N. Magini, ${ }^{99}$ L. Malgeri,${ }^{99}$ M. Mannelli, ${ }^{99}$ L. Masetti, ${ }^{99}$ F. Meijers, ${ }^{99}$ S. Mersi,${ }^{99}$ E. Meschi,${ }^{99}$ F. Moortgat,${ }^{99}$ M. Mulders ${ }^{99}$ P. Musella, ${ }^{99}$ L. Orsini,${ }^{99}$ E. Palencia Cortezon, ${ }^{99}$ E. Perez,${ }^{99}$ L. Perrozzi, ${ }^{99}$ A. Petrilli, ${ }^{99}$ G. Petrucciani,${ }^{99}$ A. Pfeiffer, ${ }^{99}$ M. Pierini, ${ }^{99}$ M. Pimiä, ${ }^{99}$ D. Piparo, ${ }^{99}$ M. Plagge, ${ }^{99}$ A. Racz,${ }^{99}$ W. Reece, ${ }^{99}$ G. Rolandi,,${ }^{99, k \mathrm{k}}$ M. Rovere, ${ }^{99}$ H. Sakulin, ${ }^{99}$ F. Santanastasio, ${ }^{99}$ C. Schäfer, ${ }^{99}$ C. Schwick, ${ }^{99}$ S. Sekmen, ${ }^{99}$ A. Sharma, ${ }^{99}$ P. Siegrist, ${ }^{99}$ P. Silva,${ }^{99}$ M. Simon, ${ }^{99}$ P. Sphicas, ${ }^{99,11}$ J. Steggemann, ${ }^{99}$ B. Stieger, ${ }^{99}$ M. Stoye, ${ }^{99}$ A. Tsirou, ${ }^{99}$ G. I. Veres, ${ }^{99, u}$ J. R. Vlimant, ${ }^{99}$ H. K. Wöhri, ${ }^{99}$ W. D. Zeuner, ${ }^{99}$ W. Bertl, ${ }^{100}$ K. Deiters,${ }^{100}$ W. Erdmann, ${ }^{100}$ K. Gabathuler ${ }^{100}$ R. Horisberger, ${ }^{100}$ Q. Ingram, ${ }^{100}$

H. C. Kaestli, ${ }^{100}$ S. König, ${ }^{100}$ D. Kotlinski ${ }^{100}$ U. Langenegger, ${ }^{100}$ D. Renker, ${ }^{100}$ T. Rohe, ${ }^{100}$ F. Bachmair, ${ }^{101}$ L. Bäni, ${ }^{101}$ L. Bianchini, ${ }^{101}$ P. Bortignon, ${ }^{101}$ M. A. Buchmann, ${ }^{101}$ B. Casal, ${ }^{101}$ N. Chanon, ${ }^{101}$ A. Deisher, ${ }^{101}$ G. Dissertori, ${ }^{101}$ M. Dittmar, ${ }^{101}$ M. Donegà, ${ }^{101}$ M. Dünser, ${ }^{101}$ P. Eller,${ }^{101}$ C. Grab,${ }^{101}$ D. Hits, ${ }^{101}$ W. Lustermann, ${ }^{101}$ B. Mangano, ${ }^{101}$ A. C. Marini, ${ }^{101}$ P. Martinez Ruiz del Arbol, ${ }^{101}$ D. Meister, ${ }^{101}$ N. Mohr, ${ }^{101}$ C. Nägeli, ${ }^{101, m m}$ P. Nef,,${ }^{101}$ F. Nessi-Tedaldi, ${ }^{101}$ F. Pandolfi, ${ }^{101}$ L. Pape,${ }^{101}$ F. Pauss, ${ }^{101}$ M. Peruzzi,${ }^{101}$ M. Quittnat, ${ }^{101}$ F. J. Ronga,${ }^{101}$ M. Rossini, ${ }^{101}$ A. Starodumov, ${ }^{101, n n}$ M. Takahashi, ${ }^{101}$ L. Tauscher, ${ }^{101, a}$ K. Theofilatos, ${ }^{101}$ D. Treille, ${ }^{101}$ R. Wallny, ${ }^{101}$ H. A. Weber, ${ }^{101}$ C. Amsler, ${ }^{102,00}$ V. Chiochia, ${ }^{102}$ A. De Cosa, ${ }^{102}$ C. Favaro, ${ }^{102}$ M. Ivova Rikova, ${ }^{102}$ B. Kilminster, ${ }^{102}$ B. Millan Mejias, ${ }^{102}$ J. Ngadiuba, ${ }^{102}$ P. Robmann, ${ }^{102}$ H. Snoek, ${ }^{102}$ S. Taroni, ${ }^{102}$ M. Verzetti, ${ }^{102}$ Y. Yang, ${ }^{102}$ M. Cardaci, ${ }^{103}$ K. H. Chen,${ }^{103}$ C. Ferro, ${ }^{103}$ C. M. Kuo, ${ }^{103}$ S. W. Li, ${ }^{103}$ W. Lin, ${ }^{103}$ Y. J. Lu, ${ }^{103}$ R. Volpe, ${ }^{103}$ S. S. Yu, ${ }^{103}$ P. Bartalini, ${ }^{104}$ P. Chang, ${ }^{104}$ Y. H. Chang, ${ }^{104}$ Y. W. Chang, ${ }^{104}$ Y. Chao, ${ }^{104}$ K. F. Chen, ${ }^{104}$ P. H. Chen, ${ }^{104}$ C. Dietz, ${ }^{104}$ U. Grundler, ${ }^{104}$ W.-S. Hou, ${ }^{104}$ Y. Hsiung, ${ }^{104}$ K. Y. Kao, ${ }^{104}$ Y. J. Lei, ${ }^{104}$ Y. F. Liu, ${ }^{104}$ R.-S. Lu, ${ }^{104}$ D. Majumder, ${ }^{104}$ E. Petrakou, ${ }^{104}$ X. Shi, ${ }^{104}$ J. G. Shiu, ${ }^{104}$ Y. M. Tzeng, ${ }^{104}$ M. Wang, ${ }^{104}$ R. Wilken, ${ }^{104}$ B. Asavapibhop, ${ }^{105}$ N. Suwonjandee, ${ }^{105}$ A. Adiguzel, ${ }^{106}$ M. N. Bakirci, ${ }^{106, p p}$ S. Cerci, ${ }^{106, q q}$ C. Dozen, ${ }^{106}$ I. Dumanoglu, ${ }^{106}$ E. Eskut, ${ }^{106}$ S. Girgis, ${ }^{106}$ G. Gokbulut, ${ }^{106}$ E. Gurpinar, ${ }^{106}$ I. Hos, ${ }^{106}$ E. E. Kangal, ${ }^{106}$

A. Kayis Topaksu, ${ }^{106}$ G. Onengut, ${ }^{106, r \mathrm{r}}$ K. Ozdemir, ${ }^{106}$ S. Ozturk, ${ }^{106, p p}$ A. Polatoz, ${ }^{106}$ K. Sogut, ${ }^{106, s s}$ D. Sunar Cerci, ${ }^{106, q q}$ B. Tali, ${ }^{106, q q}$ H. Topakli, ${ }^{106, p p}$ M. Vergili, ${ }^{106}$ I. V. Akin, ${ }^{107}$ T. Aliev,${ }^{107}$ B. Bilin, ${ }^{107}$ S. Bilmis, ${ }^{107}$ M. Deniz, ${ }^{107}$ H. Gamsizkan, ${ }^{107}$ A. M. Guler, ${ }^{107}$ G. Karapinar, ${ }^{107, t t}$ K. Ocalan, ${ }^{107}$ A. Ozpineci, ${ }^{107}$ M. Serin,${ }^{107}$ R. Sever, ${ }^{107}$ U. E. Surat, ${ }^{107}$ M. Yalvac, ${ }^{107}$ M. Zeyrek, ${ }^{107}$ E. Gülmez, ${ }^{108}$ B. Isildak, ${ }^{108, u u}$ M. Kaya, ${ }^{108, v v}$ O. Kaya, ${ }^{108, v v}$ S. Ozkorucuklu, ${ }^{108, w w}$ N. Sonmez, ${ }^{108, x x}$ H. Bahtiyar, ${ }^{109, y y}$ E. Barlas, ${ }^{109}$ K. Cankocak, ${ }^{109}$ Y. O. Günaydin, ${ }^{109, z z}$ F. I. Vardarl,${ }^{109}$ M. Yücel,${ }^{109}$ L. Levchuk, ${ }^{110}$ P. Sorokin, ${ }^{110}$ J. J. Brooke, ${ }^{111}$ E. Clement, ${ }^{111}$ D. Cussans, ${ }^{111}$ H. Flacher ${ }^{111}$ R. Frazier, ${ }^{111}$ J. Goldstein, ${ }^{111}$ M. Grimes,${ }^{111}$ G. P. Heath, ${ }^{111}$ H. F. Heath, ${ }^{111}$ J. Jacob,${ }^{111}$ L. Kreczko, ${ }^{111}$ C. Lucas, ${ }^{111}$ Z. Meng, ${ }^{111}$ D. M. Newbold, ${ }^{111 \text {,aaa }}$ S. Paramesvaran, ${ }^{111}$ A. Poll, ${ }^{111}$ S. Senkin, ${ }^{111}$ V. J. Smith, ${ }^{111}$ T. Williams, ${ }^{111}$ K. W. Bell, ${ }^{112}$ A. Belyaev, ${ }^{112, b b b}$ C. Brew, ${ }^{112}$ R. M. Brown, ${ }^{112}$ D. J. A. Cockerill, ${ }^{112}$ J. A. Coughlan, ${ }^{112}$ K. Harder, ${ }^{112}$ S. Harper, ${ }^{112}$ J. Ilic, ${ }^{112}$ E. Olaiya, ${ }^{112}$ D. Petyt, ${ }^{112}$ 
C. H. Shepherd-Themistocleous, ${ }^{112}$ A. Thea, ${ }^{112}$ I. R. Tomalin, ${ }^{112}$ W. J. Womersley, ${ }^{112}$ S. D. Worm, ${ }^{112}$ M. Baber, ${ }^{113}$ R. Bainbridge, ${ }^{113}$ O. Buchmuller, ${ }^{113}$ D. Burton, ${ }^{113}$ D. Colling, ${ }^{113}$ N. Cripps, ${ }^{113}$ M. Cutajar, ${ }^{113}$ P. Dauncey,${ }^{113}$ G. Davies, ${ }^{113}$ M. Della Negra, ${ }^{113}$ W. Ferguson, ${ }^{113}$ J. Fulcher, ${ }^{113}$ D. Futyan, ${ }^{113}$ A. Gilbert, ${ }^{113}$ A. Guneratne Bryer, ${ }^{113}$ G. Hall, ${ }^{113}$ Z. Hatherell, ${ }^{113}$ J. Hays,${ }^{113}$ G. Iles, ${ }^{113}$ M. Jarvis, ${ }^{113}$ G. Karapostoli, ${ }^{113}$ M. Kenzie,${ }^{113}$ R. Lane, ${ }^{113}$ R. Lucas, ${ }^{113 \text {,aaa }}$ L. Lyons, ${ }^{113}$ A.-M. Magnan, ${ }^{113}$ J. Marrouche, ${ }^{113}$ B. Mathias, ${ }^{113}$ R. Nandi, ${ }^{113}$ J. Nash, ${ }^{113}$ A. Nikitenko, ${ }^{113, n n}$ J. Pela,${ }^{113}$ M. Pesaresi, ${ }^{113}$ K. Petridis, ${ }^{113}$ M. Pioppi, ${ }^{11, c c c}$ D. M. Raymond, ${ }^{113}$ S. Rogerson, ${ }^{113}$ A. Rose,${ }^{113}$ C. Seez,${ }^{113}$ P. Sharp ${ }^{113, a}$ A. Sparrow, ${ }^{113}$ A. Tapper, ${ }^{113}$ M. Vazquez Acosta, ${ }^{113}$ T. Virdee, ${ }^{113}$ S. Wakefield,${ }^{113}$ N. Wardle, ${ }^{113}$ J. E. Cole,${ }^{114}$ P. R. Hobson, ${ }^{114}$ A. Khan, ${ }^{114}$ P. Kyberd,${ }^{114}$ D. Leggat, ${ }^{114}$ D. Leslie,${ }^{114}$ W. Martin ${ }^{114}$ I. D. Reid, ${ }^{114}$ P. Symonds,${ }^{114}$ L. Teodorescu, ${ }^{114}$ M. Turner, ${ }^{114}$ J. Dittmann, ${ }^{115}$ K. Hatakeyama, ${ }^{115}$ A. Kasmi, ${ }^{115}$ H. Liu, ${ }^{115}$ T. Scarborough, ${ }^{115}$ O. Charaf, ${ }^{116}$ S. I. Cooper,${ }^{116}$ C. Henderson, ${ }^{116}$ P. Rumerio, ${ }^{116}$ A. Avetisyan, ${ }^{117}$ T. Bose, ${ }^{117}$ C. Fantasia, ${ }^{117}$ A. Heister, ${ }^{117}$ P. Lawson, ${ }^{117}$ D. Lazic, ${ }^{117}$ J. Rohlf, ${ }^{117}$ D. Sperka, ${ }^{117}$ J. St. John, ${ }^{117}$ L. Sulak, ${ }^{117}$ J. Alimena, ${ }^{118}$ S. Bhattacharya, ${ }^{118}$ G. Christopher, ${ }^{118}$ D. Cutts, ${ }^{118}$

Z. Demiragli, ${ }^{118}$ A. Ferapontov, ${ }^{118}$ A. Garabedian, ${ }^{118}$ U. Heintz, ${ }^{118}$ S. Jabeen, ${ }^{118}$ G. Kukartsev, ${ }^{118}$ E. Laird, ${ }^{118}$ G. Landsberg, ${ }^{118}$ M. Luk, ${ }^{118}$ M. Narain, ${ }^{118}$ M. Segala, ${ }^{118}$ T. Sinthuprasith, ${ }^{118}$ T. Speer, ${ }^{118}$ J. Swanson, ${ }^{118}$ R. Breedon, ${ }^{119}$ G. Breto, ${ }^{119}$ M. Calderon De La Barca Sanchez, ${ }^{119}$ S. Chauhan, ${ }^{119}$ M. Chertok, ${ }^{119}$ J. Conway, ${ }^{119}$ R. Conway, ${ }^{119}$ P. T. Cox,${ }^{119}$ R. Erbacher, ${ }^{119}$ M. Gardner, ${ }^{119}$ W. Ko, ${ }^{119}$ A. Kopecky, ${ }^{119}$ R. Lander, ${ }^{119}$ T. Miceli, ${ }^{119}$ D. Pellett, ${ }^{119}$ J. Pilot, ${ }^{119}$ F. Ricci-Tam, ${ }^{119}$ B. Rutherford ${ }^{119}$ M. Searle, ${ }^{119}$ S. Shalhout, ${ }^{119}$ J. Smith ${ }^{119}$ M. Squires, ${ }^{119}$ M. Tripathi, ${ }^{119}$ S. Wilbur, ${ }^{119}$ R. Yohay, ${ }^{119}$

V. Andreev, ${ }^{120}$ D. Cline,${ }^{120}$ R. Cousins, ${ }^{120}$ S. Erhan, ${ }^{120}$ P. Everaerts, ${ }^{120}$ C. Farrell, ${ }^{120}$ M. Felcini, ${ }^{120}$ J. Hauser, ${ }^{120}$ M. Ignatenko, ${ }^{120}$ C. Jarvis, ${ }^{120}$ G. Rakness, ${ }^{120}$ P. Schlein, ${ }^{120, a}$ E. Takasugi, ${ }^{120}$ V. Valuev, ${ }^{120}$ M. Weber,${ }^{120}$ J. Babb, ${ }^{121}$ R. Clare, ${ }^{121}$ J. Ellison, ${ }^{121}$ J. W. Gary, ${ }^{121}$ G. Hanson, ${ }^{121}$ J. Heilman, ${ }^{121}$ P. Jandir, ${ }^{121}$ F. Lacroix, ${ }^{121}$ H. Liu, ${ }^{121}$ O. R. Long, ${ }^{121}$ A. Luthra ${ }^{121}$ M. Malberti, ${ }^{121}$ H. Nguyen, ${ }^{121}$ A. Shrinivas,${ }^{121}$ J. Sturdy, ${ }^{121}$ S. Sumowidagdo, ${ }^{121}$ S. Wimpenny, ${ }^{121}$ W. Andrews, ${ }^{122}$ J. G. Branson, ${ }^{122}$ G. B. Cerati, ${ }^{122}$ S. Cittolin, ${ }^{122}$ R. T. D’ Agnolo, ${ }^{122}$ D. Evans, ${ }^{122}$ A. Holzner, ${ }^{122}$ R. Kelley, ${ }^{122}$ D. Kovalskyi, ${ }^{122}$ M. Lebourgeois, ${ }^{122}$ J. Letts, ${ }^{122}$ I. Macneill, ${ }^{122}$ S. Padhi, ${ }^{122}$ C. Palmer, ${ }^{122}$ M. Pieri, ${ }^{122}$ M. Sani, ${ }^{122}$ V. Sharma, ${ }^{122}$ S. Simon, ${ }^{122}$ E. Sudano, ${ }^{122}$ M. Tadel, ${ }^{122}$ Y. Tu, ${ }^{122}$ A. Vartak, ${ }^{122}$ S. Wasserbaech,${ }^{122, \text { ddd }}$ F. Würthwein, ${ }^{122}$ A. Yagil, ${ }^{122}$ J. Yoo, ${ }^{122}$ D. Barge, ${ }^{123}$ C. Campagnari, ${ }^{123}$ T. Danielson, ${ }^{123}$ K. Flowers, ${ }^{123}$ P. Geffert, ${ }^{123}$ C. George,${ }^{123}$ F. Golf, ${ }^{123}$ J. Incandela, ${ }^{123}$ C. Justus, ${ }^{123}$ R. Magaña Villalba, ${ }^{123}$ N. Mccoll, ${ }^{123}$ V. Pavlunin, ${ }^{123}$ J. Richman, ${ }^{123}$ R. Rossin, ${ }^{123}$ D. Stuart, ${ }^{123}$ W. To, ${ }^{123}$ C. West, ${ }^{123}$ A. Apresyan, ${ }^{124}$ A. Bornheim, ${ }^{124}$ J. Bunn, ${ }^{124}$ Y. Chen, ${ }^{124}$ E. Di Marco, ${ }^{124}$ J. Duarte, ${ }^{124}$ D. Kcira, ${ }^{124}$ A. Mott, ${ }^{124}$ H. B. Newman,${ }^{124}$ C. Pena,${ }^{124}$ C. Rogan, ${ }^{124}$ M. Spiropulu, ${ }^{124}$ V. Timciuc, ${ }^{124}$ R. Wilkinson, ${ }^{124}$ S. Xie, ${ }^{124}$ R. Y. Zhu, ${ }^{124}$ V. Azzolini, ${ }^{125}$ A. Calamba, ${ }^{125}$ R. Carroll, ${ }^{125}$ T. Ferguson, ${ }^{125}$ Y. Iiyama, ${ }^{125}$ D. W. Jang, ${ }^{125}$ M. Paulini, ${ }^{125}$ J. Russ, ${ }^{125}$ H. Vogel, ${ }^{125}$ I. Vorobiev, ${ }^{125}$ J. P. Cumalat, ${ }^{126}$ B. R. Drell, ${ }^{126}$ W. T. Ford, ${ }^{126}$ A. Gaz, ${ }^{126}$ E. Luiggi Lopez, ${ }^{126}$ U. Nauenberg, ${ }^{126}$ J. G. Smith, ${ }^{126}$ K. Stenson, ${ }^{126}$ K. A. Ulmer, ${ }^{126}$ S. R. Wagner, ${ }^{126}$ J. Alexander, ${ }^{127}$ A. Chatterjee, ${ }^{127}$ N. Eggert, ${ }^{127}$ L. K. Gibbons, ${ }^{127}$ W. Hopkins, ${ }^{127}$ A. Khukhunaishvili, ${ }^{127}$ B. Kreis, ${ }^{127}$ N. Mirman, ${ }^{127}$ G. Nicolas Kaufman, ${ }^{127}$ J. R. Patterson, ${ }^{127}$ A. Ryd,${ }^{127}$ E. Salvati, ${ }^{127}$ W. Sun ${ }^{127}$ W. D. Teo, ${ }^{127}$ J. Thom, ${ }^{127}$ J. Thompson, ${ }^{127}$ J. Tucker, ${ }^{127}$ Y. Weng, ${ }^{127}$

L. Winstrom, ${ }^{127}$ P. Wittich, ${ }^{127}$ D. Winn, ${ }^{128}$ S. Abdullin, ${ }^{129}$ M. Albrow, ${ }^{129}$ J. Anderson, ${ }^{129}$ G. Apollinari, ${ }^{129}$

L. A. T. Bauerdick, ${ }^{129}$ A. Beretvas, ${ }^{129}$ J. Berryhill, ${ }^{129}$ P. C. Bhat, ${ }^{129}$ K. Burkett, ${ }^{129}$ J. N. Butler, ${ }^{129}$ V. Chetluru, ${ }^{129}$ H. W. K. Cheung, ${ }^{129}$ F. Chlebana, ${ }^{129}$ S. Cihangir, ${ }^{129}$ V. D. Elvira, ${ }^{129}$ I. Fisk, ${ }^{129}$ J. Freeman, ${ }^{129}$ Y. Gao, ${ }^{129}$ E. Gottschalk, ${ }^{129}$ L. Gray, ${ }^{129}$ D. Green, ${ }^{129}$ O. Gutsche, ${ }^{129}$ D. Hare, ${ }^{129}$ R. M. Harris, ${ }^{129}$ J. Hirschauer, ${ }^{129}$ B. Hooberman, ${ }^{129}$ S. Jindariani, ${ }^{129}$ M. Johnson, ${ }^{129}$ U. Joshi, ${ }^{129}$ K. Kaadze, ${ }^{129}$ B. Klima, ${ }^{129}$ S. Kwan, ${ }^{129}$ J. Linacre, ${ }^{129}$ D. Lincoln, ${ }^{129}$ R. Lipton, ${ }^{129}$ J. Lykken, ${ }^{129}$

K. Maeshima, ${ }^{129}$ J. M. Marraffino, ${ }^{129}$ V. I. Martinez Outschoorn, ${ }^{129}$ S. Maruyama ${ }^{129}$ D. Mason,${ }^{129}$ P. McBride, ${ }^{129}$ K. Mishra, ${ }^{129}$ S. Mrenna, ${ }^{129}$ Y. Musienko, ${ }^{129, \text { eee }}$ S. Nahn, ${ }^{129}$ C. Newman-Holmes, ${ }^{129}$ V. O’Dell, ${ }^{129}$ O. Prokofyev, ${ }^{129}$ N. Ratnikova ${ }^{129}$ E. Sexton-Kennedy, ${ }^{129}$ S. Sharma ${ }^{129}$ W. J. Spalding, ${ }^{129}$ L. Spiegel, ${ }^{129}$ L. Taylor, ${ }^{129}$ S. Tkaczyk, ${ }^{129}$ N. V. Tran, ${ }^{129}$ L. Uplegger, ${ }^{129}$ E. W. Vaandering, ${ }^{129}$ R. Vidal, ${ }^{129}$ J. Whitmore, ${ }^{129}$ W. Wu, ${ }^{129}$ F. Yang, ${ }^{129}$ J. C. Yun, ${ }^{129}$ D. Acosta, ${ }^{130}$ P. Avery, ${ }^{130}$ D. Bourilkov, ${ }^{130}$ T. Cheng, ${ }^{130}$ S. Das, ${ }^{130}$ M. De Gruttola, ${ }^{130}$ G. P. Di Giovanni, ${ }^{130}$ D. Dobur, ${ }^{130}$ R. D. Field, ${ }^{130}$ M. Fisher, ${ }^{130}$ Y. Fu, ${ }^{130}$ I. K. Furic, ${ }^{130}$ J. Hugon, ${ }^{130}$ B. Kim,${ }^{130}$ J. Konigsberg, ${ }^{130}$ A. Korytov, ${ }^{130}$ A. Kropivnitskaya, ${ }^{130}$ T. Kypreos, ${ }^{130}$ J. F. Low, ${ }^{130}$ K. Matchev, ${ }^{130}$ P. Milenovic, ${ }^{130, f f}$ G. Mitselmakher, ${ }^{130}$ L. Muniz ${ }^{130}$ A. Rinkevicius, ${ }^{130}$ L. Shchutska, ${ }^{130}$ N. Skhirtladze, ${ }^{130}$ M. Snowball, ${ }^{130}$ J. Yelton, ${ }^{130}$ M. Zakaria, ${ }^{130}$ V. Gaultney, ${ }^{131}$ S. Hewamanage, ${ }^{131}$ S. Linn, ${ }^{131}$ P. Markowitz, ${ }^{131}$ G. Martinez, ${ }^{131}$ J. L. Rodriguez, ${ }^{131}$ T. Adams, ${ }^{132}$ A. Askew, ${ }^{132}$ J. Bochenek, ${ }^{132}$ J. Chen, ${ }^{132}$ B. Diamond, ${ }^{132}$ J. Haas, ${ }^{132}$ S. Hagopian, ${ }^{132}$ V. Hagopian, ${ }^{132}$ K. F. Johnson, ${ }^{132}$ H. Prosper, ${ }^{132}$ V. Veeraraghavan, ${ }^{132}$ M. Weinberg, ${ }^{132}$ M. M. Baarmand, ${ }^{133}$ B. Dorney, ${ }^{133}$ M. Hohlmann, ${ }^{133}$ H. Kalakhety, ${ }^{133}$ F. Yumiceva, ${ }^{133}$ M. R. Adams, ${ }^{134}$ L. Apanasevich, ${ }^{134}$ V. E. Bazterra, ${ }^{134}$ R. R. Betts, ${ }^{134}$ I. Bucinskaite, ${ }^{134}$ R. Cavanaugh, ${ }^{134}$ O. Evdokimov, ${ }^{134}$ 
L. Gauthier, ${ }^{134}$ C. E. Gerber, ${ }^{134}$ D. J. Hofman, ${ }^{134}$ S. Khalatyan, ${ }^{134}$ P. Kurt, ${ }^{134}$ D. H. Moon, ${ }^{134}$ C. O’Brien, ${ }^{134}$ C. Silkworth, ${ }^{134}$ P. Turner, ${ }^{134}$ N. Varelas, ${ }^{134}$ U. Akgun, ${ }^{135}$ E. A. Albayrak, ${ }^{135, y y}$ B. Bilki, ${ }^{135, g g g}$ W. Clarida, ${ }^{135}$ K. Dilsiz, ${ }^{135}$ F. Duru, ${ }^{135}$ J.-P. Merlo, ${ }^{135}$ H. Mermerkaya, ${ }^{135, \text { hhh }}$ A. Mestvirishvili, ${ }^{135}$ A. Moeller, ${ }^{135}$ J. Nachtman, ${ }^{135}$ H. Ogul, ${ }^{135}$ Y. Onel, ${ }^{135}$ F. Ozok, ${ }^{135, y y}$ S. Sen, ${ }^{135}$ P. Tan, ${ }^{135}$ E. Tiras, ${ }^{135}$ J. Wetzel, ${ }^{135}$ T. Yetkin, ${ }^{135, \text { iii }}$ K. Yi, ${ }^{135}$ B. A. Barnett, ${ }^{136}$ B. Blumenfeld, ${ }^{136}$ S. Bolognesi, ${ }^{136}$ D. Fehling, ${ }^{136}$ A. V. Gritsan, ${ }^{136}$ P. Maksimovic, ${ }^{136}$ C. Martin,${ }^{136}$ M. Swartz,${ }^{136}$ A. Whitbeck, ${ }^{136}$ P. Baringer, ${ }^{137}$ A. Bean, ${ }^{137}$ G. Benelli, ${ }^{137}$ R. P. Kenny IIII, ${ }^{137}$ M. Murray, ${ }^{137}$ D. Noonan, ${ }^{137}$ S. Sanders, ${ }^{137}$ J. Sekaric, ${ }^{137}$ R. Stringer, ${ }^{137}$ Q. Wang, ${ }^{137}$ J. S. Wood, ${ }^{137}$ A. F. Barfuss, ${ }^{138}$ I. Chakaberia, ${ }^{138}$ A. Ivanov, ${ }^{138}$ S. Khalil, ${ }^{138}$ M. Makouski, ${ }^{138}$ Y. Maravin, ${ }^{138}$ L. K. Saini, ${ }^{138}$ S. Shrestha, ${ }^{138}$ I. Svintradze, ${ }^{138}$ J. Gronberg, ${ }^{139}$ D. Lange, ${ }^{139}$ F. Rebassoo, ${ }^{139}$ D. Wright, ${ }^{139}$ A. Baden, ${ }^{140}$ B. Calvert, ${ }^{140}$ S. C. Eno, ${ }^{140}$ J. A. Gomez, ${ }^{140}$ N. J. Hadley, ${ }^{140}$ R. G. Kellogg, ${ }^{140}$ T. Kolberg, ${ }^{140}$ Y. Lu, ${ }^{140}$ M. Marionneau, ${ }^{140}$ A. C. Mignerey, ${ }^{140}$ K. Pedro, ${ }^{140}$ A. Skuja, ${ }^{140}$ J. Temple, ${ }^{140}$ M. B. Tonjes, ${ }^{140}$ S. C. Tonwar, ${ }^{140}$ A. Apyan, ${ }^{141}$ R. Barbieri, ${ }^{141}$ G. Bauer, ${ }^{141}$ W. Busza,${ }^{141}$ I. A. Cali, ${ }^{141}$ M. Chan,${ }^{141}$ L. Di Matteo, ${ }^{141}$ V. Dutta, ${ }^{141}$ G. Gomez Ceballos, ${ }^{141}$ M. Goncharov ${ }^{141}$ D. Gulhan, ${ }^{141}$ M. Klute, ${ }^{141}$ Y. S. Lai, ${ }^{141}$ Y.-J. Lee, ${ }^{141}$ A. Levin, ${ }^{141}$ P. D. Luckey, ${ }^{141}$ T. Ma ${ }^{141}$ C. Paus, ${ }^{141}$ D. Ralph, ${ }^{141}$ C. Roland,${ }^{141}$ G. Roland,${ }^{141}$ G. S. F. Stephans, ${ }^{141}$ F. Stöckli, ${ }^{141}$ K. Sumorok,${ }^{141}$ D. Velicanu, ${ }^{141}$ J. Veverka, ${ }^{141}$ B. Wyslouch, ${ }^{141}$ M. Yang, ${ }^{141}$ A. S. Yoon, ${ }^{141}$ M. Zanetti,${ }^{141}$ V. Zhukova, ${ }^{141}$ B. Dahmes, ${ }^{142}$ A. De Benedetti, ${ }^{142}$ A. Gude, ${ }^{142}$ S. C. Kao,${ }^{142}$ K. Klapoetke, ${ }^{142}$ Y. Kubota, ${ }^{142}$ J. Mans, ${ }^{142}$ N. Pastika, ${ }^{142}$ R. Rusack, ${ }^{142}$ A. Singovsky, ${ }^{142}$ N. Tambe,${ }^{142}$ J. Turkewitz, ${ }^{142}$ J. G. Acosta, ${ }^{143}$ L. M. Cremaldi, ${ }^{143}$ R. Kroeger, ${ }^{143}$ S. Oliveros, ${ }^{143}$ L. Perera, ${ }^{143}$ R. Rahmat, ${ }^{143}$ D. A. Sanders, ${ }^{143}$ D. Summers, ${ }^{143}$ E. Avdeeva, ${ }^{144}$ K. Bloom, ${ }^{144}$ S. Bose,${ }^{144}$ D. R. Claes, ${ }^{144}$ A. Dominguez, ${ }^{144}$ R. Gonzalez Suarez, ${ }^{144}$ J. Keller, ${ }^{144}$ I. Kravchenko, ${ }^{144}$ J. Lazo-Flores, ${ }^{144}$ S. Malik, ${ }^{144}$ F. Meier, ${ }^{144}$ G. R. Snow, ${ }^{144}$ J. Dolen, ${ }^{145}$ A. Godshalk, ${ }^{145}$ I. Iashvili, ${ }^{145}$ S. Jain, ${ }^{145}$ A. Kharchilava, ${ }^{145}$ A. Kumar, ${ }^{145}$ S. Rappoccio, ${ }^{145}$ Z. Wan, ${ }^{145}$ G. Alverson, ${ }^{146}$ E. Barberis, ${ }^{146}$ D. Baumgartel, ${ }^{146}$ M. Chasco, ${ }^{146}$ J. Haley, ${ }^{146}$ A. Massironi, ${ }^{146}$ D. Nash, ${ }^{146}$ T. Orimoto, ${ }^{146}$ D. Trocino, ${ }^{146}$ D. Wood, ${ }^{146}$ J. Zhang, ${ }^{146}$ A. Anastassov, ${ }^{147}$ K. A. Hahn, ${ }^{147}$ A. Kubik, ${ }^{147}$ L. Lusito, ${ }^{147}$ N. Mucia, ${ }^{147}$ N. Odell, ${ }^{147}$ B. Pollack, ${ }^{147}$ A. Pozdnyakov, ${ }^{147}$ M. Schmitt, ${ }^{147}$ S. Stoynev, ${ }^{147}$ K. Sung, ${ }^{147}$ M. Velasco,${ }^{147}$ S. Won ${ }^{147}$ D. Berry, ${ }^{148}$ A. Brinkerhoff, ${ }^{148}$ K. M. Chan ${ }^{148}$ A. Drozdetskiy, ${ }^{148}$ M. Hildreth, ${ }^{148}$ C. Jessop ${ }^{148}$ D. J. Karmgard, ${ }^{148}$ J. Kolb, ${ }^{148}$ K. Lannon, ${ }^{148}$ W. Luo, ${ }^{148}$ S. Lynch, ${ }^{148}$ N. Marinelli, ${ }^{148}$ D. M. Morse ${ }^{148}$ T. Pearson, ${ }^{148}$ M. Planer, ${ }^{148}$ R. Ruchti, ${ }^{148}$ J. Slaunwhite, ${ }^{148}$ N. Valls, ${ }^{148}$ M. Wayne, ${ }^{148}$ M. Wolf, ${ }^{148}$ L. Antonelli, ${ }^{149}$ B. Bylsma, ${ }^{149}$ L. S. Durkin, ${ }^{149}$ S. Flowers,${ }^{149}$ C. Hill, ${ }^{149}$ R. Hughes,${ }^{149}$ K. Kotov, ${ }^{149}$ T. Y. Ling, ${ }^{149}$ D. Puigh, ${ }^{149}$ M. Rodenburg, ${ }^{149}$ G. Smith, ${ }^{149}$ C. Vuosalo, ${ }^{149}$ B. L. Winer, ${ }^{149}$ H. Wolfe, ${ }^{149}$ H. W. Wulsin, ${ }^{149}$ E. Berry, ${ }^{150}$ P. Elmer, ${ }^{150}$ V. Halyo, ${ }^{150}$ P. Hebda, ${ }^{150}$ J. Hegeman, ${ }^{150}$ A. Hunt, ${ }^{150}$ P. Jindal,${ }^{150}$ S. A. Koay, ${ }^{150}$ P. Lujan, ${ }^{150}$ D. Marlow, ${ }^{150}$ T. Medvedeva, ${ }^{150}$ M. Mooney, ${ }^{150}$ J. Olsen, ${ }^{150}$ P. Piroué, ${ }^{150}$ X. Quan, ${ }^{150}$ A. Raval,${ }^{150}$ H. Saka, ${ }^{150}$ D. Stickland, ${ }^{150}$ C. Tully, ${ }^{150}$ J. S. Werner, ${ }^{150}$ S. C. Zenz, ${ }^{150}$ A. Zuranski, ${ }^{150}$ E. Brownson, ${ }^{151}$ A. Lopez, ${ }^{151}$ H. Mendez, ${ }^{151}$ J. E. Ramirez Vargas, ${ }^{151}$ E. Alagoz, ${ }^{152}$ D. Benedetti, ${ }^{152}$ G. Bolla, ${ }^{152}$ D. Bortoletto, ${ }^{152}$ M. De Mattia, ${ }^{152}$ A. Everett, ${ }^{152}$ Z. Hu, ${ }^{152}$ M. Jones, ${ }^{152}$ K. Jung, ${ }^{152}$ M. Kress, ${ }^{152}$ N. Leonardo, ${ }^{152}$ D. Lopes Pegna, ${ }^{152}$ V. Maroussov, ${ }^{152}$ P. Merkel, ${ }^{152}$ D. H. Miller, ${ }^{152}$ N. Neumeister ${ }^{152}$ B. C. Radburn-Smith, ${ }^{152}$ I. Shipsey, ${ }^{152}$ D. Silvers, ${ }^{152}$ A. Svyatkovskiy, ${ }^{152}$ F. Wang, ${ }^{152}$ W. Xie, ${ }^{152}$ L. Xu, ${ }^{152}$ H. D. Yoo, ${ }^{152}$ J. Zablocki, ${ }^{152}$ Y. Zheng, ${ }^{152}$ N. Parashar, ${ }^{153}$ A. Adair, ${ }^{154}$ B. Akgun, ${ }^{154}$ K. M. Ecklund, ${ }^{154}$ F. J. M. Geurts, ${ }^{154}$ W. Li, ${ }^{154}$ B. Michlin, ${ }^{154}$ B. P. Padley, ${ }^{154}$ R. Redjimi, ${ }^{154}$ J. Roberts, ${ }^{154}$ J. Zabel, ${ }^{154}$ B. Betchart, ${ }^{155}$ A. Bodek,${ }^{155}$ R. Covarelli, ${ }^{155}$ P. de Barbaro, ${ }^{155}$ R. Demina, ${ }^{155}$ Y. Eshaq, ${ }^{155}$ T. Ferbel, ${ }^{155}$ A. Garcia-Bellido, ${ }^{155}$ P. Goldenzweig, ${ }^{155}$ J. Han, ${ }^{155}$ A. Harel, ${ }^{155}$ D. C. Miner,${ }^{155}$ G. Petrillo, ${ }^{155}$ D. Vishnevskiy, ${ }^{155}$ M. Zielinski, ${ }^{155}$ A. Bhatti, ${ }^{156}$ R. Ciesielski, ${ }^{156}$ L. Demortier, ${ }^{156}$ K. Goulianos, ${ }^{156}$ G. Lungu, ${ }^{156}$ S. Malik, ${ }^{156}$ C. Mesropian, ${ }^{156}$ S. Arora, ${ }^{157}$ A. Barker, ${ }^{157}$ J. P. Chou, ${ }^{157}$ C. Contreras-Campana ${ }^{157}$ E. Contreras-Campana, ${ }^{157}$ D. Duggan, ${ }^{157}$ D. Ferencek,${ }^{157}$ Y. Gershtein, ${ }^{157}$ R. Gray, ${ }^{157}$ E. Halkiadakis, ${ }^{157}$ D. Hidas, ${ }^{157}$ A. Lath,${ }^{157}$ S. Panwalkar, ${ }^{157}$ M. Park,${ }^{157}$ R. Patel,${ }^{157}$ V. Rekovic, ${ }^{157}$ J. Robles,${ }^{157}$ S. Salur, ${ }^{157}$ S. Schnetzer, ${ }^{157}$ C. Seitz, ${ }^{157}$ S. Somalwar, ${ }^{157}$ R. Stone, ${ }^{157}$ S. Thomas, ${ }^{157}$ P. Thomassen, ${ }^{157}$ M. Walker, ${ }^{157}$ K. Rose ${ }^{158}$ S. Spanier, ${ }^{158}$ Z. C. Yang, ${ }^{158}$ A. York, ${ }^{158}$ O. Bouhali, ${ }^{159, j j j}$ R. Eusebi, ${ }^{159}$ W. Flanagan, ${ }^{159}$ J. Gilmore, ${ }^{159}$ T. Kamon, ${ }^{159, k k k}$ V. Khotilovich, ${ }^{159}$ V. Krutelyov, ${ }^{159}$ R. Montalvo, ${ }^{159}$ I. Osipenkov, ${ }^{159}$ Y. Pakhotin, ${ }^{159}$ A. Perloff, ${ }^{159}$ J. Roe, ${ }^{159}$ A. Safonov, ${ }^{159}$ T. Sakuma, ${ }^{159}$ I. Suarez, ${ }^{159}$ A. Tatarinov, ${ }^{159}$ D. Toback, ${ }^{159}$ N. Akchurin, ${ }^{160}$ C. Cowden,,${ }^{160}$ J. Damgov, ${ }^{160}$ C. Dragoiu, ${ }^{160}$ P. R. Dudero, ${ }^{160}$ K. Kovitanggoon, ${ }^{160}$ S. Kunori, ${ }^{160}$ S. W. Lee, ${ }^{160}$ T. Libeiro, ${ }^{160}$ I. Volobouev, ${ }^{160}$ E. Appelt, ${ }^{161}$ A. G. Delannoy, ${ }^{161}$ S. Greene, ${ }^{161}$ A. Gurrola, ${ }^{161}$ W. Johns, ${ }^{161}$ C. Maguire, ${ }^{161}$ Y. Mao, ${ }^{161}$ A. Melo, ${ }^{161}$ M. Sharma, ${ }^{161}$ P. Sheldon, ${ }^{161}$ B. Snook, ${ }^{161}$ S. Tuo, ${ }^{161}$ J. Velkovska, ${ }^{161}$ M. W. Arenton, ${ }^{162}$ S. Boutle, ${ }^{162}$ B. Cox, ${ }^{162}$ B. Francis, ${ }^{162}$ J. Goodell, ${ }^{162}$ R. Hirosky, ${ }^{162}$ A. Ledovskoy, ${ }^{162}$ C. Lin, ${ }^{162}$ C. Neu, ${ }^{162}$ J. Wood,${ }^{162}$ S. Gollapinni, ${ }^{163}$ R. Harr, ${ }^{163}$

P. E. Karchin, ${ }^{163}$ C. Kottachchi Kankanamge Don, ${ }^{163}$ P. Lamichhane, ${ }^{163}$ A. Sakharov, ${ }^{163}$ D. A. Belknap,${ }^{164}$ L. Borrello, ${ }^{164}$ D. Carlsmith, ${ }^{164}$ M. Cepeda, ${ }^{164}$ S. Dasu, ${ }^{164}$ S. Duric, ${ }^{164}$ E. Friis, ${ }^{164}$ M. Grothe, ${ }^{164}$ R. Hall-Wilton, ${ }^{164}$ M. Herndon, ${ }^{164}$ 
A. Hervé, ${ }^{164}$ P. Klabbers, ${ }^{164}$ J. Klukas, ${ }^{164}$ A. Lanaro, ${ }^{164}$ R. Loveless, ${ }^{164}$ A. Mohapatra, ${ }^{164}$ I. Ojalvo, ${ }^{164}$ T. Perry, ${ }^{164}$ G. A. Pierro, ${ }^{164}$ G. Polese, ${ }^{164}$ I. Ross, ${ }^{164}$ T. Sarangi, ${ }^{164}$ A. Savin ${ }^{164}$ and W. H. Smith ${ }^{164}$

(CMS Collaboration)

\author{
${ }^{1}$ Yerevan Physics Institute, Yerevan, Armenia \\ ${ }^{2}$ Institut für Hochenergiephysik der OeAW, Wien, Austria \\ ${ }^{3}$ National Centre for Particle and High Energy Physics, Minsk, Belarus \\ ${ }^{4}$ Universiteit Antwerpen, Antwerpen, Belgium \\ ${ }^{5}$ Vrije Universiteit Brussel, Brussel, Belgium \\ ${ }^{6}$ Université Libre de Bruxelles, Bruxelles, Belgium \\ ${ }^{7}$ Ghent University, Ghent, Belgium \\ ${ }^{8}$ Université Catholique de Louvain, Louvain-la-Neuve, Belgium \\ ${ }^{9}$ Université de Mons, Mons, Belgium \\ ${ }^{10}$ Centro Brasileiro de Pesquisas Fisicas, Rio de Janeiro, Brazil \\ ${ }^{11}$ Universidade do Estado do Rio de Janeiro, Rio de Janeiro, Brazil \\ ${ }^{12 a}$ Universidade Estadual Paulista, São Paulo, Brazil \\ ${ }^{12 \mathrm{~b}}$ Universidade Federal do ABC, São Paulo, Brazil \\ ${ }^{13}$ Institute for Nuclear Research and Nuclear Energy, Sofia, Bulgaria \\ ${ }^{14}$ University of Sofia, Sofia, Bulgaria \\ ${ }^{15}$ Institute of High Energy Physics, Beijing, China \\ ${ }^{16}$ State Key Laboratory of Nuclear Physics and Technology, Peking University, Beijing, China \\ ${ }^{17}$ Universidad de Los Andes, Bogota, Colombia \\ ${ }^{18}$ Technical University of Split, Split, Croatia \\ ${ }^{19}$ University of Split, Split, Croatia \\ ${ }^{20}$ Institute Rudjer Boskovic, Zagreb, Croatia \\ ${ }^{21}$ University of Cyprus, Nicosia, Cyprus \\ ${ }^{22}$ Charles University, Prague, Czech Republic \\ ${ }^{23}$ Academy of Scientific Research and Technology of the Arab Republic of Egypt, \\ Egyptian Network of High Energy Physics, Cairo, Egypt \\ ${ }^{24}$ National Institute of Chemical Physics and Biophysics, Tallinn, Estonia \\ ${ }^{25}$ Department of Physics, University of Helsinki, Helsinki, Finland \\ ${ }^{26}$ Helsinki Institute of Physics, Helsinki, Finland \\ ${ }^{27}$ Lappeenranta University of Technology, Lappeenranta, Finland \\ ${ }^{28}$ DSM/IRFU, CEA/Saclay, Gif-sur-Yvette, France \\ ${ }^{29}$ Laboratoire Leprince-Ringuet, Ecole Polytechnique, IN2P3-CNRS, Palaiseau, France \\ ${ }^{30}$ Institut Pluridisciplinaire Hubert Curien, Université de Strasbourg, Université de Haute Alsace Mulhouse, \\ CNRS/IN2P3, Strasbourg, France
}

${ }^{31}$ Centre de Calcul de l'Institut National de Physique Nucleaire et de Physique des Particules, CNRS/IN2P3, Villeurbanne, France

${ }^{32}$ Université de Lyon, Université Claude Bernard Lyon 1, CNRS-IN2P3, Institut de Physique Nucléaire de Lyon, Villeurbanne, France

${ }^{33}$ Institute of High Energy Physics and Informatization, Tbilisi State University, Tbilisi, Georgia

${ }^{34}$ RWTH Aachen University, I. Physikalisches Institut, Aachen, Germany

${ }^{35}$ RWTH Aachen University, III. Physikalisches Institut A, Aachen, Germany

${ }^{36}$ RWTH Aachen University, III. Physikalisches Institut B, Aachen, Germany

${ }^{37}$ Deutsches Elektronen-Synchrotron, Hamburg, Germany

${ }^{38}$ University of Hamburg, Hamburg, Germany

${ }^{39}$ Institut für Experimentelle Kernphysik, Karlsruhe, Germany

${ }^{40}$ Institute of Nuclear and Particle Physics (INPP), NCSR Demokritos, Aghia Paraskevi, Greece

${ }^{41}$ University of Athens, Athens, Greece

${ }^{42}$ University of Ioánnina, Ioánnina, Greece

${ }^{43}$ Wigner Research Centre for Physics, Budapest, Hungary

${ }^{44}$ Institute of Nuclear Research ATOMKI, Debrecen, Hungary

${ }^{45}$ University of Debrecen, Debrecen, Hungary

${ }^{46}$ National Institute of Science Education and Research, Bhubaneswar, India

${ }^{47}$ Panjab University, Chandigarh, India

${ }^{48}$ University of Delhi, Delhi, India

${ }^{49}$ Saha Institute of Nuclear Physics, Kolkata, India

${ }^{50}$ Bhabha Atomic Research Centre, Mumbai, India

${ }^{51}$ Tata Institute of Fundamental Research - EHEP, Mumbai, India 


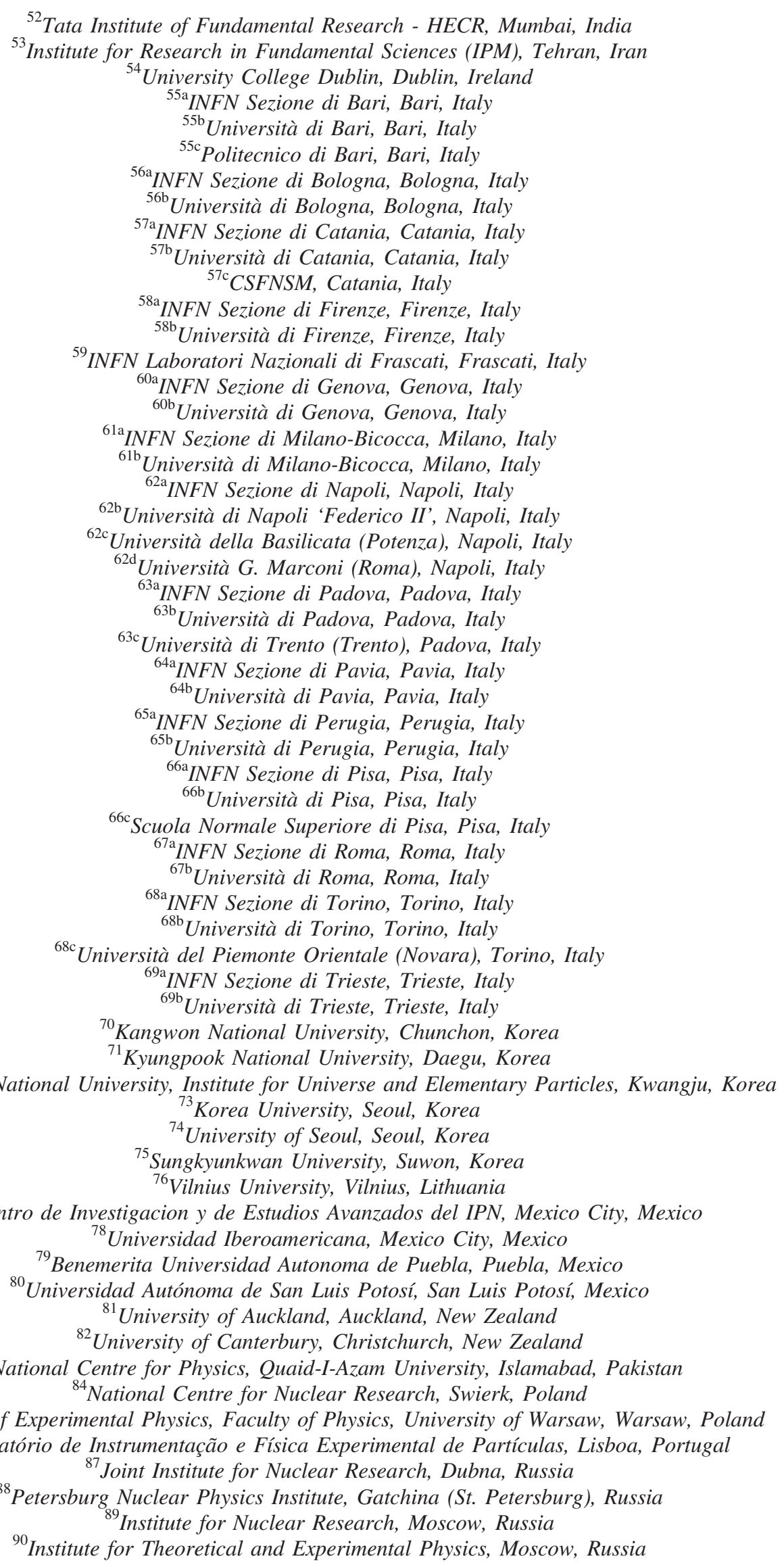


${ }^{91}$ P.N. Lebedev Physical Institute, Moscow, Russia

${ }^{92}$ Skobeltsyn Institute of Nuclear Physics, Lomonosov Moscow State University, Moscow, Russia

${ }^{93}$ State Research Center of Russian Federation, Institute for High Energy Physics, Protvino, Russia

${ }^{94}$ University of Belgrade, Faculty of Physics and Vinca Institute of Nuclear Sciences, Belgrade, Serbia

${ }^{95}$ Centro de Investigaciones Energéticas Medioambientales y Tecnológicas (CIEMAT), Madrid, Spain

${ }^{96}$ Universidad Autónoma de Madrid, Madrid, Spain

${ }^{97}$ Universidad de Oviedo, Oviedo, Spain

${ }^{98}$ Instituto de Física de Cantabria (IFCA), CSIC-Universidad de Cantabria, Santander, Spain

${ }^{99}$ CERN, European Organization for Nuclear Research, Geneva, Switzerland

${ }^{100}$ Paul Scherrer Institut, Villigen, Switzerland

${ }^{101}$ Institute for Particle Physics, ETH Zurich, Zurich, Switzerland

${ }^{102}$ Universität Zürich, Zurich, Switzerland

${ }^{103}$ National Central University, Chung-Li, Taiwan

${ }^{104}$ National Taiwan University (NTU), Taipei, Taiwan

${ }^{105}$ Chulalongkorn University, Bangkok, Thailand

${ }^{106}$ Cukurova University, Adana, Turkey

${ }^{107}$ Middle East Technical University, Physics Department, Ankara, Turkey

${ }^{108}$ Bogazici University, Istanbul, Turkey

${ }^{109}$ Istanbul Technical University, Istanbul, Turkey

${ }^{110}$ National Scientific Center, Kharkov Institute of Physics and Technology, Kharkov, Ukraine

${ }^{111}$ University of Bristol, Bristol, United Kingdom

${ }^{112}$ Rutherford Appleton Laboratory, Didcot, United Kingdom

${ }^{113}$ Imperial College, London, United Kingdom

${ }^{114}$ Brunel University, Uxbridge, United Kingdom

${ }^{115}$ Baylor University, Waco, USA

${ }^{116}$ The University of Alabama, Tuscaloosa, USA

${ }^{117}$ Boston University, Boston, USA

${ }^{118}$ Brown University, Providence, USA

${ }^{119}$ University of California, Davis, Davis, USA

${ }^{120}$ University of California, Los Angeles, USA

${ }^{121}$ University of California, Riverside, Riverside, USA

${ }^{122}$ University of California, San Diego, La Jolla, USA

${ }^{123}$ University of California, Santa Barbara, Santa Barbara, USA

${ }^{124}$ California Institute of Technology, Pasadena, USA

${ }^{125}$ Carnegie Mellon University, Pittsburgh, USA

${ }^{126}$ University of Colorado at Boulder, Boulder, USA

${ }^{127}$ Cornell University, Ithaca, USA

${ }^{128}$ Fairfield University, Fairfield, USA

${ }^{129}$ Fermi National Accelerator Laboratory, Batavia, USA

${ }^{130}$ University of Florida, Gainesville, USA

${ }^{131}$ Florida International University, Miami, USA

${ }^{132}$ Florida State University, Tallahassee, USA

${ }^{133}$ Florida Institute of Technology, Melbourne, USA

${ }^{134}$ University of Illinois at Chicago (UIC), Chicago, USA

${ }^{135}$ The University of Iowa, Iowa City, USA

${ }^{136}$ Johns Hopkins University, Baltimore, USA

${ }^{137}$ The University of Kansas, Lawrence, USA

${ }^{138}$ Kansas State University, Manhattan, USA

${ }^{139}$ Lawrence Livermore National Laboratory, Livermore, USA

${ }^{140}$ University of Maryland, College Park, USA

${ }^{141}$ Massachusetts Institute of Technology, Cambridge, USA

${ }^{142}$ University of Minnesota, Minneapolis, USA

${ }^{143}$ University of Mississippi, Oxford, USA

${ }^{144}$ University of Nebraska-Lincoln, Lincoln, USA

${ }^{145}$ State University of New York at Buffalo, Buffalo, USA

${ }^{146}$ Northeastern University, Boston, USA

${ }^{147}$ Northwestern University, Evanston, USA

${ }^{148}$ University of Notre Dame, Notre Dame, USA

${ }^{149}$ The Ohio State University, Columbus, USA

${ }^{150}$ Princeton University, Princeton, USA 


\author{
${ }^{151}$ University of Puerto Rico, Mayaguez, USA \\ ${ }^{152}$ Purdue University, West Lafayette, USA \\ ${ }^{153}$ Purdue University Calumet, Hammond, USA \\ ${ }^{154}$ Rice University, Houston, USA \\ ${ }^{155}$ University of Rochester, Rochester, USA \\ ${ }^{156}$ The Rockefeller University, New York, USA \\ ${ }^{157}$ Rutgers, The State University of New Jersey, Piscataway, USA \\ ${ }^{158}$ University of Tennessee, Knoxville, USA \\ ${ }^{159}$ Texas A\&M University, College Station, USA \\ ${ }^{160}$ Texas Tech University, Lubbock, USA \\ ${ }^{161}$ Vanderbilt University, Nashville, USA \\ ${ }^{162}$ University of Virginia, Charlottesville, USA \\ ${ }^{163}$ Wayne State University, Detroit, USA \\ ${ }^{164}$ University of Wisconsin, Madison, USA
}

${ }^{\mathrm{a}}$ Deceased.

${ }^{\mathrm{b}}$ Also at Vienna University of Technology, Vienna, Austria.

${ }^{c}$ Also at CERN, European Organization for Nuclear Research, Geneva, Switzerland.

${ }^{\mathrm{d}}$ Also at Institut Pluridisciplinaire Hubert Curien, Université de Strasbourg, Université de Haute Alsace Mulhouse, CNRS/IN2P3, Strasbourg, France.

${ }^{\mathrm{e}}$ Also at National Institute of Chemical Physics and Biophysics, Tallinn, Estonia.

${ }^{\mathrm{f}}$ Also at Skobeltsyn Institute of Nuclear Physics, Lomonosov Moscow State University, Moscow, Russia.

${ }^{\mathrm{g}}$ Also at Universidade Estadual de Campinas, Campinas, Brazil.

${ }^{\mathrm{h}}$ Also at California Institute of Technology, Pasadena, USA.

${ }^{\mathrm{i}}$ Also at Laboratoire Leprince-Ringuet, Ecole Polytechnique, IN2P3-CNRS, Palaiseau, France.

${ }^{\mathrm{j}}$ Also at Zewail City of Science and Technology, Zewail, Egypt.

${ }^{\mathrm{k}}$ Also at Suez Canal University, Suez, Egypt.

${ }^{1}$ Also at Cairo University, Cairo, Egypt.

${ }^{\mathrm{m}}$ Also at Fayoum University, El-Fayoum, Egypt.

${ }^{\mathrm{n}}$ Also at British University in Egypt, Cairo, Egypt.

${ }^{\circ}$ Now at Ain Shams University, Cairo, Egypt.

${ }^{\mathrm{P}}$ Also at Université de Haute Alsace, Mulhouse, France.

${ }^{\mathrm{q}}$ Also at Joint Institute for Nuclear Research, Dubna, Russia.

${ }^{\mathrm{r}}$ Also at Brandenburg University of Technology, Cottbus, Germany.

${ }^{\mathrm{s}}$ Also at The University of Kansas, Lawrence, USA.

${ }^{\mathrm{t}}$ Also at Institute of Nuclear Research ATOMKI, Debrecen, Hungary.

uAlso at Eötvös Loránd University, Budapest, Hungary.

${ }^{v}$ Also at Tata Institute of Fundamental Research - EHEP, Mumbai, India.

${ }^{\mathrm{w}}$ Also at Tata Institute of Fundamental Research - HECR, Mumbai, India.

${ }^{\mathrm{x}}$ Now at King Abdulaziz University, Jeddah, Saudi Arabia.

${ }^{y}$ Also at University of Visva-Bharati, Santiniketan, India.

${ }^{\mathrm{z}}$ Also at University of Ruhuna, Matara, Sri Lanka.

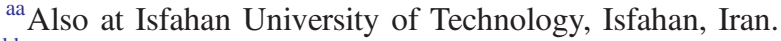

${ }^{\mathrm{bb}}$ Also at Sharif University of Technology, Tehran, Iran.

${ }^{\mathrm{cc}}$ Also at Plasma Physics Research Center, Science and Research Branch, Islamic Azad University, Tehran, Iran.

${ }^{\mathrm{dd}}$ Also at Università degli Studi di Siena, Siena, Italy.

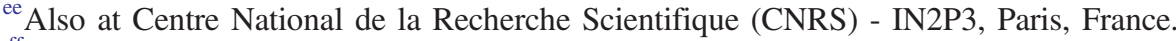

${ }^{\mathrm{ff}}$ Also at Purdue University, West Lafayette, USA.

${ }^{g g}$ Also at Universidad Michoacana de San Nicolas de Hidalgo, Morelia, Mexico.

${ }^{\text {hh }}$ Also at National Centre for Nuclear Research, Swierk, Poland.

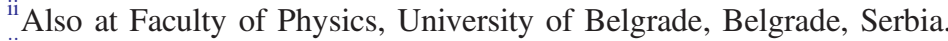

${ }^{\mathrm{jj}}$ Also at Facoltà Ingegneria, Università di Roma, Roma, Italy.

${ }^{\mathrm{kk}}$ Also at Scuola Normale e Sezione dell'INFN, Pisa, Italy.

${ }^{11}$ Also at University of Athens, Athens, Greece.

${ }^{\mathrm{mm}}$ Also at Paul Scherrer Institut, Villigen, Switzerland.

${ }^{n n}$ Also at Institute for Theoretical and Experimental Physics, Moscow, Russia.

${ }^{\circ o}$ Also at Albert Einstein Center for Fundamental Physics, Bern, Switzerland.

${ }^{\mathrm{pp}}$ Also at Gaziosmanpasa University, Tokat, Turkey.

${ }^{\mathrm{qq}}$ Also at Adiyaman University, Adiyaman, Turkey.

${ }^{\mathrm{rr}}$ Also at Cag University, Mersin, Turkey. 


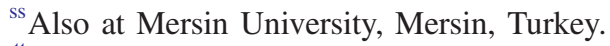

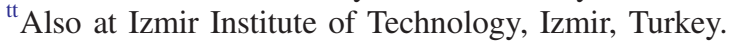

${ }^{u u}$ Also at Ozyegin University, Istanbul, Turkey.

${ }^{\mathrm{vv}}$ Also at Kafkas University, Kars, Turkey.

${ }^{w w}$ Also at Suleyman Demirel University, Isparta, Turkey.

${ }^{\mathrm{xx}}$ Also at Ege University, Izmir, Turkey.

${ }^{\text {yy }}$ Also at Mimar Sinan University, Istanbul, Istanbul, Turkey.

${ }^{z z}$ Also at Kahramanmaras Sütcü Imam University, Kahramanmaras, Turkey.

${ }^{\text {aaa }}$ Also at Rutherford Appleton Laboratory, Didcot, United Kingdom.

${ }^{b b b}$ Also at School of Physics and Astronomy, University of Southampton, Southampton, United Kingdom.

${ }^{c c c}$ Also at INFN Sezione di Perugia, Università di Perugia, Perugia, Italy.

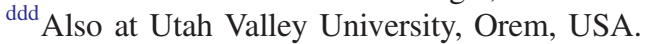

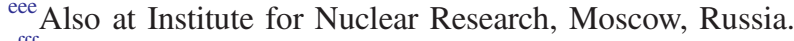

${ }^{\mathrm{fff}}$ Also at University of Belgrade, Faculty of Physics and Vinca Institute of Nuclear Sciences, Belgrade, Serbia.

${ }^{\text {ggg }}$ Also at Argonne National Laboratory, Argonne, USA.

${ }^{\text {hhh }}$ Also at Erzincan University, Erzincan, Turkey.

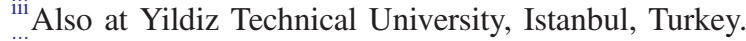

${ }^{j j j}$ Also at Texas A\&M University at Qatar, Doha, Qatar.

${ }^{k k k}$ Also at Kyungpook National University, Daegu, Korea. 